\title{
Comparison of MSL RCS jet computations with flow visualization and velocimetry
}

\author{
C.T. Johansen ${ }^{1}$ \\ University of Calgary, Calgary, $A B, T 2 N 1 N 4$ \\ L. Novak ${ }^{2}$, B. Bathel ${ }^{3}$, S.W. Ashcraft ${ }^{4}$, P.M. Danehy ${ }^{5}$ \\ NASA Langley Research Center, Hampton VA, 23681-2199
}

\begin{abstract}
Numerical predictions of the Mars Science Laboratory (MSL) reaction control system (RCS) jets interacting with a Mach 10 hypersonic flow are compared to experimental nitric oxide (NO) planar laser-induced fluorescence (PLIF) data. The steady Reynolds Averaged Navier Stokes (RANS) equations using the Baldwin-Barth one-equation turbulence model were solved using the OVERFLOW code. The experimental PLIF data used for comparison consists of qualitative two-dimensional visualization images, qualitative reconstructed threedimensional flow structures, and quantitative two-dimensional distributions of streamwise velocity. Through modeling of the PLIF signal equation, computational flow images (CFI) were produced and directly compared to the qualitative PLIF data. Post processing of the experimental and simulation data enabled the jet trajectory to be extracted for a more quantitative comparison. The two-dimensional velocity fields were reconstructed through interpolation of a series of single-component velocity profiles. Each distribution of singlecomponent velocity was obtained using molecular tagging velocimetry (MTV). After validating the numerical model, the numerical solution was further examined to gain insight into hypersonic jet-in-crossflow interaction. Future NO-PLIF experiments are proposed based on this analysis.
\end{abstract}

\section{Introduction}

$\mathrm{T}$ HE Mars Science Laboratory (MSL) vehicle recently launched by NASA in November, 2011 is expected to arrive and enter the Martian atmosphere in August, 2012. Derived from the Viking mission design, the MSL is a lifting, capsule-type entry vehicle with a heat shield cone half angle of 70 degrees. The vehicle will undergo an ambitious entry, descent, and landing (EDL) process, which includes the execution of a series of bank reversal maneuvers using the vehicle's reaction control system (RCS) jets. ${ }^{1}$ As shown in side view of the MSL vehicle in Fig. 1, the RCS jet pairs are distributed and orientated on the aftbody to provide pitch, roll, and yaw moments to the vehicle. The aerodynamic maneuvers will improve the down-range and cross-range landing accuracy while the strategic firing of RCS jets will counteract aerodynamic instabilities and atmospheric dispersions. A significant experimental and computational effort, motivated by the NASA Engineering and Safety Center (NESC), has been undertaken to understand and quantify any large and unexpected aerodynamic interferences that might occur when the RCS jets are activated. The NESC study objectives were to validate the CFD models to show that there were no serious discrepancies in the aero/RCS interactions that had been predicted and to advance the CFD aero/RCS predictive capabilities. There is an expected interaction between the reaction control system (RCS) jets and the capsule wake in addition to interaction between individual RCS jets. ${ }^{1-4}$ The hypersonic flow field consists of a strong bow shock wave ahead of the capsule forebody, a supersonic expansion fan around the capsule shoulder, and

${ }^{1}$ Assistant Professor, Mechanical and Manufacturing Engineering, Member AIAA.

${ }^{2}$ Project Engineer, Analytical Mechanics Associates Inc., Hampton VA 23666; Currently Software Engineer, Sukra Helitek Inc., Ames IA 50010, Member AIAA.

${ }^{3}$ Graduate Coop Student in Advanced Sensing and Optical Measurement Branch, MS 493; also Graduate Student, University of Virginia. AIAA Student Member

${ }^{4}$ USRP Student, Advance Sensing and Optical Measurement Branch, MS 493, also Student at University of Kentucky. AIAA Student Member

${ }^{5}$ Research Scientist, Advanced Sensing and Optical Measurement Branch, MS 493. AIAA Associate Fellow. 
a large subsonic separated region in the capsule wake. Recompression shock waves redirect the outerflow downstream of the capsule wake. The capsule has a radial center-of-gravity offset that causes the vehicle trim at an angle-of-attack, providing lift for maneuvering. The RCS jets are used to perform bank maneuvers, changing the pointing direction of the lift vector to fly a guided entry. The under-expanded RCS jets interact with both attached supersonic cross-flows and separated subsonic recirculation zones depending on the jet location and vehicle angle of attack. Further details of the vehicle aerodynamics during re-entry are discussed in the literature. ${ }^{3,4}$

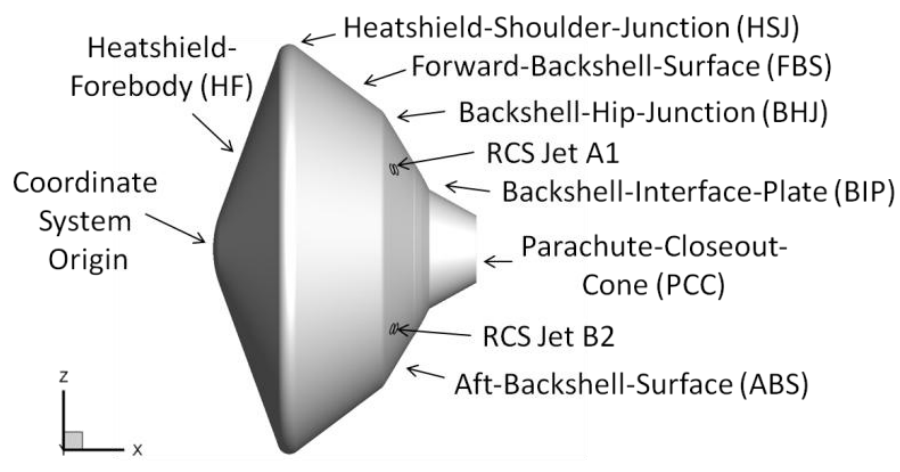

Figure 1. MSL aeroshell configuration and nomenclature. The origin for the Cartesian coordinate system $(x$, $y, z)$ is located on the nose of the forebody heat-shield.

There is a strong reliance on computational fluid dynamics (CFD) to aid in the design of capsule-type entry vehicles because of the high costs of ground-based tests and the impracticality of flight tests in the Martian atmosphere. Although the computational resources available to simulate the high-speed aerodynamics are improving, there is still a strong dependence on simplified numerical models to predict the transport of mass, momentum, and energy. Turbulence modeling, for example, needs to be included in the simulation of the MSL vehicle aerodynamics because of the high Reynolds numbers that are expected to occur during the peak heating loads of atmospheric entry. ${ }^{5}$ High Reynolds numbers occur because of MSL's relatively large capsule diameter and high ballistic coefficient in comparison to previous vehicles designs. ${ }^{6}$ Although direct numerical simulation (DNS) offers the most accurate means to predict this type of flow, the computational resources available are insufficient to resolve the entire turbulence spectrum. By partially modeling the turbulence spectrum, large eddy simulation (LES) offers a compromise between DNS and Reynolds Averaged Navier Stokes (RANS) simulations. Although LES alleviates the computational load requirements, the technique still requires high spatial and temporal resolution and long run times to collect statistics. By modeling the entire turbulence spectrum, RANS simulations can provide steady-state solutions of the mean variables with relatively short turn-around times. A recent computational study of the MSL RCS jet flow field was performed using detached eddy simulation (DES), which is a hybrid LES/RANS model. ${ }^{7}$ In that work, LES was used to predict the unsteady separated flow in the capsule wake and the RANS-type model was used to predict the flow near the vehicle surface. That work provided insight into the unsteady nature of the RCS jet-crossflow interaction. Each configuration, however, took approximately 66 hours to complete using each of the 240 cores of the University of Minnesota Supercomputing Institute's SGI Altix XE Linux Cluster. Based on the long run-times, the technique is not yet practical for design optimization or for parametric studies. Therefore, the current strategy for future entry vehicle design is still to use RANS-based models for prediction and rely on a robust and comprehensive validation against trusted ground-based experiments. ${ }^{2,3}$ As a result of the NESC initiative, recent detailed experimental data of flow separation and jet interaction associated with the MSL vehicle are now available for a rigorous CFD validation. ${ }^{4}$ Validation is crucial, especially for RANS-type models, since they have difficulty in predicting turbulent flows with streamline curvature or flow separation. ${ }^{9}$

In high-speed aerodynamics, non-intrusive laser diagnostic measurements are preferred over conventional probebased techniques to build a validation dataset. Laser-based measurements are preferred because it is often unfeasible to place a probe inside a hypersonic flow field since the mere presence of a physical object can artificially distort the flow and contaminate the results. Furthermore, some laser diagnostic techniques, such as planar laser-induced fluorescence (PLIF), are able to extract highly resolved and spatially correlated data, which is preferred over traversing a single-point measurement probe. Quantitative PLIF techniques can also be used to extract flow variables such as velocity, temperature and species concentrations needed for direct comparison to CFD. A major problem that occurs with numerical model validation using quantitative laser diagnostics is that the experimental 
techniques are usually restricted to narrow operating conditions. ${ }^{10}$ With computational flow imaging (CFI), both the fluid dynamics and spectroscopic process are modeled to produce simulated PLIF images, which can then be compared to experimental PLIF images. This strategy offers comprehensive model validation and simplifies the laser diagnostic technique and experimental setup. Some early work using the CFI technique to compare simulation predictions to experimental PLIF images for high-speed aerodynamic applications can be found in Refs. 11 and 12.

In the present work, numerical predictions of the MSL RCS jet flow field using the OVERFLOW code and the Baldwin-Barth one-equation turbulence model are compared to an experimental PLIF validation dataset. The experimental validation dataset was produced from a wind tunnel test in NASA Langley Research Center's 31-inch Mach 10 facility in $2010 .{ }^{4}$ In that study, nitric oxide (NO) gas was supplied to both the RCS jet plenum and to the sting that held the $1 / 291 / 2$ scale model of the MSL flight vehicle. This facilitated NO-PLIF visualization of both the capsule wake and the RCS jets for jet-on and jet-off conditions. A CFI model was applied to the numerical solution to enable comparisons to the experimental flow visualization data. Three dimensional distributions of PLIF signal intensity near the RCS jets were reconstructed from the original experimental two-dimensional PLIF images. Similarly, three-dimensional jet structures were extracted from the simulated PLIF field for comparison. Further post-processing and analysis of both the experimental and simulated flow visualization data have been performed to make comparisons of the RCS jet trajectory. To supplement flow visualization, molecular tagging velocimetry (MTV) was used to map the aftbody velocity field. After demonstrating the accuracy of the simulation, the numerical solution was further examined to gain insight into hypersonic jet-in-crossflow interaction and to further understand the limits of the PLIF measurement technique. Based on that analysis, future experiments using the NO PLIF technique are proposed.

\section{Wind Tunnel and Model Experimental Setup}

The experimental data used for the validation was performed in NASA Langley Research Center's 31-Inch Mach 10 Air Tunnel facility in $2010 .{ }^{4}$ By electrically heating compressed air, the facility is able to provide Mach 10 flow through the 31 -inch square test section for approximately 90 seconds. ${ }^{13}$ The test section has optical access through two large windows located on the top and bottom tunnel walls. A third smaller window is located on the side wall opposite to the model injection system. Each window transmits ultraviolet light, which allows the projection of the laser sheet through the top window into the test section. Digital images of the NO fluorescence were acquired through the side window.

Figure 2 shows the side view of the 1/291/2 scale titanium MSL entry capsule model. As shown, the capsule is at an angle of attack (AoA) of -20 degrees with respect to the flow. This angle corresponds to a horizontal sting. A water-cooled 5-component force and moment balance was located inside the capsule and aligned with the model's center of gravity (obviating the need for axial force to perform moment transfers from the balance moment reference point to the capsule center of gravity). The balance cabling, thermocouple cabling, and $\mathrm{N}_{2} / \mathrm{NO}$ plumbing were protected from aerodynamic heating during the $\sim 90$ second runtimes by a protective shroud. The sting AoA was adjusted during the run using a motorized control system. For most of the runs, NO was seeded into the flow only through the B2 RCS jet pair (Fig. 1). The RCS jet fluid was seeded with 2-5\% NO, with the remaining gas being $\mathrm{N}_{2}$. This allowed observation of the shape, structure and trajectory of the RCS jets. In some runs, the RCS jets were not operated. Instead, pure (100\%) NO was seeded into the flow through a gap between the sting and the shroud placed over the sting. As a result, the NO was entrained in the separated wake flow behind the capsule, allowing for the visualization of the wake's shape and size. For the MTV measurement in the wake with the B2 RCS jet pair on, a 2$5 \% \mathrm{NO} / \mathrm{N}_{2}$ mixture was seeded into both the jet plenum and model-sting junction. Reference 4 should be consulted for further details of the experimental setup and run conditions. 


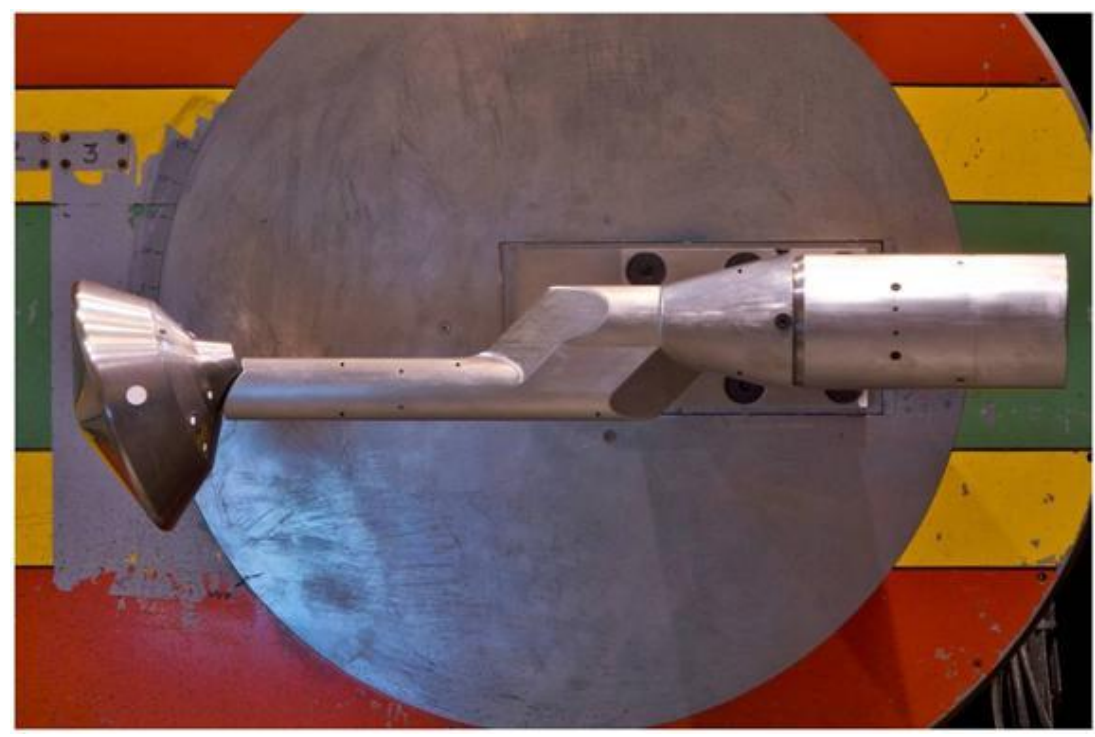

Figure 2. Side view photograph of MSL model, sting with protective heat shroud, and tunnel side wall. Reproduced from Ref. 4.

\section{PLIF Visualization and Velocity Measurement Techniques}

The NO PLIF system uses an ultraviolet laser sheet to interrogate a slice in the flow containing seeded NO. This UV light sheet excited fluorescence from the seeded NO molecules. The fluorescence was detected by the highspeed PI-MAX II intensified CCD digital camera. The laser sheet could be swept through the flow, thereby visualizing different cross sections of the flow. In post-processing, these different spatial measurements could be recombined to show the three dimensional structure of the wake flow and RCS jets. Details of the measurement system used in this experiment, as well as the experimental procedure used, can be found in Refs. 14 and 15 .

Streamwise velocity measurements were made by measuring the displacement of a series of lines aligned vertically with respect to the freestream flow in a streamwise plane. The lines were formed by passing a portion of the laser sheet through a 50-mm-long LaserOptik diffusion-welded lens array. The lens array consisted of $251 \mathrm{~m}$ focal length cylindrical lenses that focused the sheet into 25 collimated beams. The beams were then passed into the flow and excited fluorescence in the NO molecules. A Cooke DiCAM-Pro dual-frame intensified camera was used to probe the molecules at the instant they were tagged (initial image) and $500 \mathrm{~ns}$ after excitation (delayed image). The initial and delayed images were compared to determine the streamwise $\left(\boldsymbol{V}_{\boldsymbol{x}}\right)$ component of velocity. A detailed discussion of the experimental methodology can be found in Ref. 4. A detailed discussion of the velocity and uncertainty analysis can be found in Ref. 16. In this paper, modifications have been made to the methodology described in Ref. 16 for calculating the estimated time delay and timing uncertainty for sequential images. A difference of less than $1 \%$ in both measured mean velocity and uncertainty the in mean velocity is expected using these modifications versus using the methodology of Ref. 15. The two-dimensional velocity fields were reconstructed through interpolation of the single-component velocity distributions. The flow visualization and velocity data were imported into Tecplot $360^{\circledR}$ for comparison with the CFD results.

\section{Computational Fluid Dynamics Simulation}

CFD simulations of the flow field were performed with the OVERFLOW (the OVERset grid FLOW solver) Version 2.2 code ${ }^{17,18}$ The simulations solved the compressible Reynolds Averaged Navier Stokes equations. The Baldwin-Barth one-equation model was used for turbulence closure. The implicit time advancing scheme used was the Symmetric Successive Over-Relaxation (SSOR) method. The third-order HLLC (Harten, Lax, van Leer, and Einfeldt) numerical flux function with the Van Albada limiter was used. Three grid-sequencing levels were used to accelerate steady-state convergence. Solutions were considered converged when the first three significant figures of the component (i.e. backshell) mean aerodynamic force coefficient did not change for approximately 2000 iterations.

The domain geometry is approximately three capsule diameters in the $y$-direction, four capsule diameters in the $z$ - direction, and four and a half capsule diameters in the $x$-direction. The far-field boundaries are modeled with a characteristic inflow/outflow boundary condition. The gas composition at the inflow boundary is air. The inflow 
stagnation pressure, stagnation temperature, and Mach number are $9.11 \mathrm{MPa}, 1032 \mathrm{~K}$, and 9.94, respectively. The solid walls are modeled with a no-slip, adiabatic boundary condition with an estimated $\mathrm{y}^{+}$of one using a flat plate approximation. The RCS nozzle was modeled back to the plenum, where total pressure and temperature conditions from the test are enforced. At the plenum boundary, pure $\mathrm{N}_{2}$ with a stagnation pressure of $689.5 \mathrm{kPa}$ and stagnation temperature of $300 \mathrm{~K}$ was specified. The domain was discretized using overset structured grid blocks whose interfaces are interpolated via stencils defined using the Pegasus5 code. Approximately 50 million nodes were used in the simulation.

The perfect gas law was used in the simulations. In the analysis, a CFD-computed map of the streamwise component of velocity was compared directly with experimental velocity measurements. Two additional steps were required to compare the PLIF flow visualization data with CFD predictions of the RCS jet plumes. First, the RCS jet gas was assumed to be $5 \% \mathrm{NO} / 95 \% \mathrm{~N}_{2}$ instead of pure $\mathrm{N}_{2}$. To clarify, pure $\mathrm{N} 2$ was used in the simulations and $\mathrm{NO}$ was added later in post-processing by assuming a 5\% molar fraction with respect to N2. Chemistry was assumed to be frozen. Second, the CFD solution was used to compute a theoretical PLIF image using the polynomial method described in Ref. 19. Such computed PLIF images were compared with experimental PLIF intensity images using iso-contours in Tecplot.

\section{Validation Results}

Below, direct comparisons between CFD and the experimental PLIF data are made for three different types of measurements: separated wake flow measurements, RCS jet structure and trajectory measurements, and streamwise velocity measurements. Comparison of the separated wake flow and RCS jet structure are qualitative while comparisons of the jet-trajectory and streamwise velocity are quantitative.

\section{A. Wake Flow Visualization without RCS Jets}

Figure 3a shows an NO fluorescence image of the separated wake behind an MSL model vehicle obtained in the previous study of the vehicle's aerodynamics. ${ }^{4}$ In this configuration, pure NO was seeded into the flow through a gap between the sting and the shroud placed over the sting. The reversed flow in the separated wake convected the NO gas upstream of the Parachute Closeout Cone (PCC) near the separation point. The laser sheet interrogated the flow vertically from top to bottom and was positioned on the model centerline. Although both the laser position and model AoA were parameters that were varied in the experiments, all comparisons in this current study are evaluated at a model AoA of $-20^{\circ}$. In the previous work the PLIF data were visualized using the Virtual Diagnostics Interface (ViDI) method, which allows for accurate placement of the processed image data relative to the MSL model. In the current study, Tecplot was used as the visualization tool. ViDI, however, was used to prepare the PLIF data before being imported into Tecplot. Figure $2 \mathrm{~b}$ shows the separated wake at the same conditions described above, but with stream traces overlaid from the OVERFLOW CFD solution. Unlike ViDI, which displays a series of individual images, Tecplot displays a true three-dimensional reconstruction of all of the PLIF data. The image shown in Figure $3 \mathrm{~b}$ is a slice of experimental PLIF data extracted from the three-dimensional reconstruction to show the original twodimensional distribution of the PLIF signal. To clarify, after the two-dimensional images were loaded into an interpolated three-dimensional Tecplot data file, a two-dimensional slice was extracted to produce the image. Although the original two-dimensional images could be used to produce Fig. 3b, the process described above was implemented to verify the accuracy of the three-dimensional data files that were constructed in Tecplot. Both the field of view and image resolution were reduced to produce manageable three-dimensional Tecplot files to be loaded onto a desktop PC. As shown in this qualitative comparison, the location and size of primary and secondary recirculation zones agree well with streamline predictions from the OVERFLOW code. 
a)

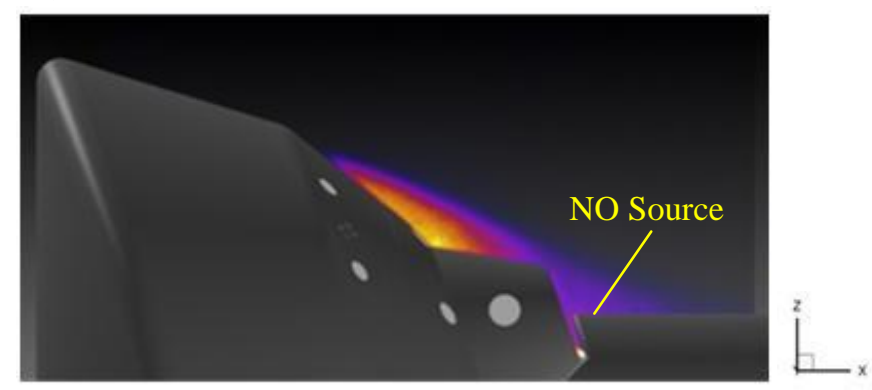

b)

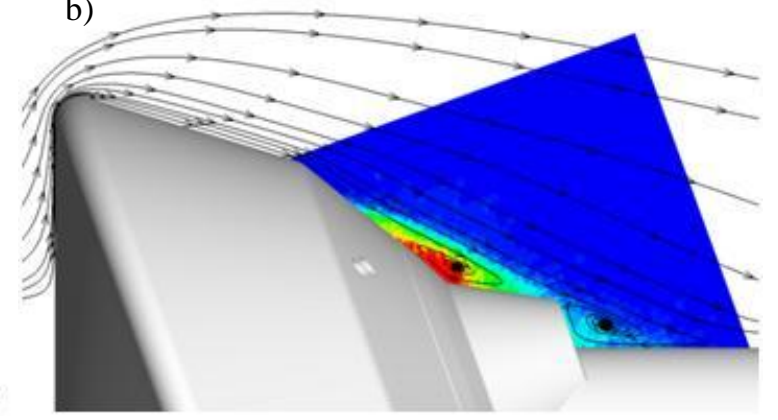

Figure 3. Visualization of the separated wake flow at model centerline using pure NO seeding (jet off): a) Experimental PLIF distribution shown using ViDI method, reproduced from Ref. 4; b) Experimental PLIF contour plot with overlaid streamlines predicted by OVERFLOW.

By scanning the laser sheet in the spanwise $(-y)$ direction, the three-dimensionality of the wake can be observed. Figure 4 shows an isometric view of the MSL model with two-dimensional contour maps of the PLIF signal at three separate spanwise locations. A series of stream traces predicted by OVERFLOW are overlaid onto the figure. Similar to the flow separation that occurs from the backshell-interface-plate (BIP) near the model centerline $(y=0$ $\mathrm{mm}$ ), NO becomes entrained into a larger recirculation zone that extends from the PCC to the heatshield-shoulderjunction (HSJ) near $z=0 \mathrm{~mm}$. Separation does not occur at the top HSJ near the model centerline $(y=0 \mathrm{~mm})$ since the model is pitched forward. This creates a relatively shallow angle between the freestream flow and model surface allowing the flow to remain attached. Discussion of the three-dimensional wake shape in relation to the effective surface angle is discussed in past work. ${ }^{4}$

a)

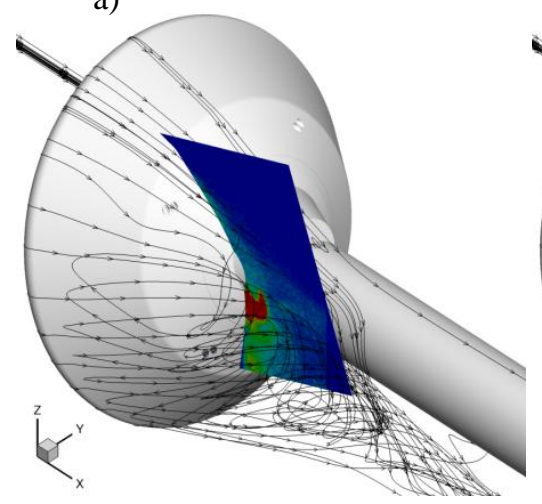

b)

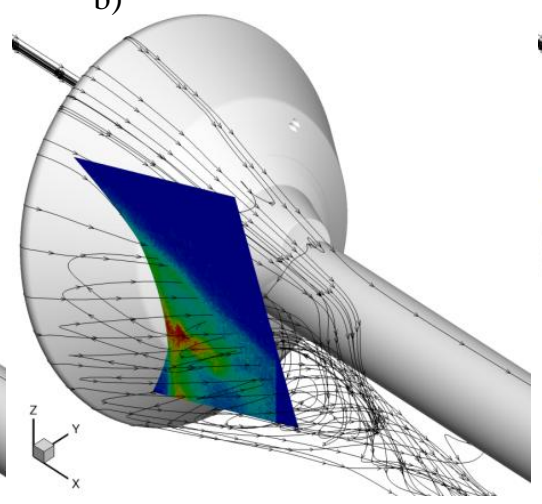

c)

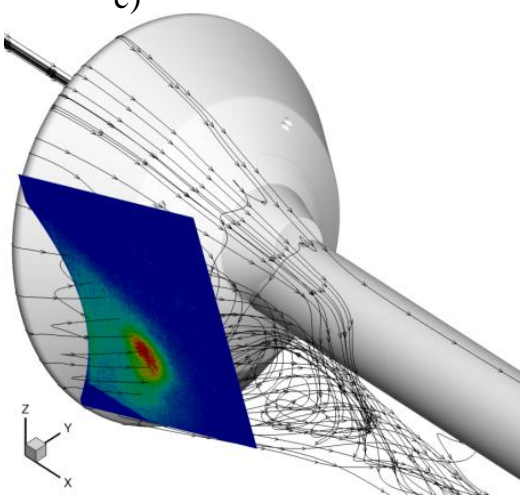

Figure 4. 3D visualization of the separated wake flow through translation of laser sheet: a) $y=-20 \mathrm{~mm}$; b) $y=-46 \mathrm{~mm}$; c) $y=-63 \mathrm{~mm}$. Stream traces predicted by OVERFLOW.

Note that the experimental PLIF data do not extend far enough in the downstream $(+x)$ or in the vertical $(-z)$ directions to fully capture the size of the separated wake. Restrictions in the camera field of view and laser sheet dimensions prohibited these measurements. Where the experimental data are available, the OVERFLOW prediction of stream traces agrees well with the outer boundary of PLIF signal. Near the sting, the predicted stream traces indicate that the large recirculation zone forms into a trailing vortex. Although vorticity in the near field is dominated by the $z$ - and $y$-component of vorticity, redistribution to $x$-component vorticity occurs in the far field. Although the PLIF data are not available downstream of the main separated region, NO is expected to exist further downstream because of entrainment into the trailing vortices.

Figure 5 shows a three-dimensional iso-surface of the PLIF signal with the simulated stream traces. To compare the overall shape of the separated region, side, top, back, and isometric views are shown in Fig. 5. The vertical and 
streamwise limits of the PLIF data field-of-view are evident from the images. For example, full separation is predicted on the lower forward-backshell surface (FBS) and over the aft-backshell surface (ABS), but PLIF data are not available for comparison (denoted by the hard edges in the iso-surface data). For the data that are available, there is good agreement between the experimental and computed separated shape. The simulation predicts a dip in the separated shape that is confirmed by the experimental data. The location of the dip is indicated by the red arrow in the isometric view of the figure. This location corresponds to the interface that forms between the counter-rotating vortices in the far field. The stream traces just below (-y direction) the model centerline, as shown in the $x-y$ view, roll up into the counter-clockwise vortex ( $y-z$ view). In contrast, the remaining lower streamlines in the $x-y$ view rollup into the stronger clockwise rotating vortex. The clockwise rotating vortex is identifiable on the left side in the $y-z$ view. Note that the presence of the sting is expected to influence the trajectory and strength of the counterrotating vortex pair. As a result, caution must be exercised when extrapolating these observations to the wake of the MSL flight vehicle. Unfortunately, the presence of the sting also blocked the projection of the laser sheet through the lower counter-clockwise vortex and therefore prevented study of the NO entrainment into this predicted vortex. These observations, however, are relevant for model validation purposes since the sting was included in the simulations.
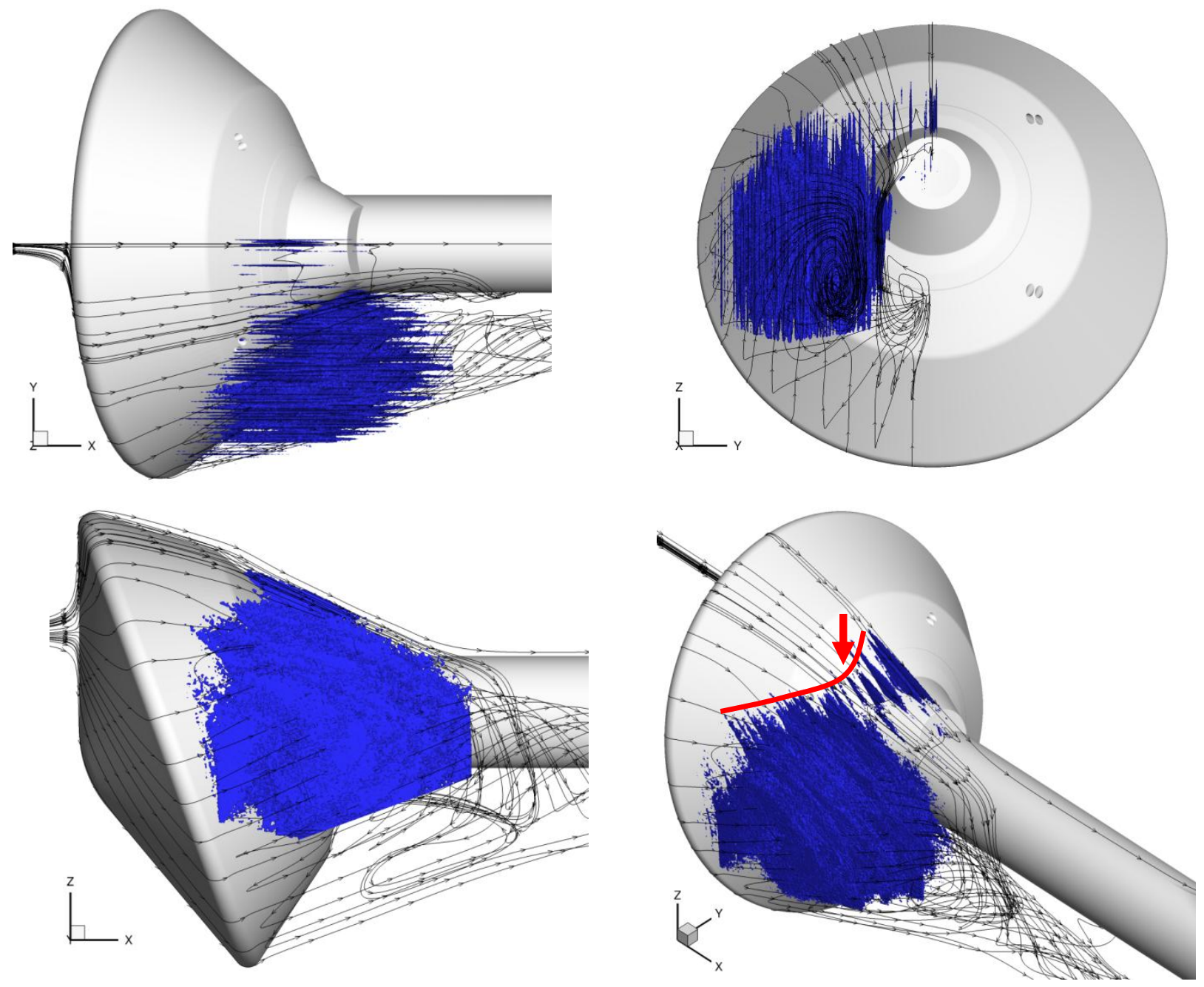

Figure 5. 3D comparison of the separated wake. PLIF data is shown as iso-surface. Stream traces predicted by OVERFLOW. Red arrow indicates location of dip in separated profile as described in text. 


\section{B. RCS Jet-Flow Interaction}

The interaction of the RCS jets with the capsule wake is of interest both for aerodynamic and aerothermodynamic considerations. Figure 6 shows RCS B2 jet visualization with the MSL model oriented at $-20^{\circ}$ AoA. The image in Figure 6a was produced using the ViDI technique. Similar to the wake-seeding technique, the RCS jets are seeded with NO and the laser sheet is scanned in the spanwise direction. As a result, the threedimensional structure and overall trajectory of the jet is visualized. Figure $6 \mathrm{~b}$ shows the same data visualized as an iso-surface of the PLIF signal $\left(S_{f}=20\right)$ using Tecplot. Note that values of $S_{f}$ are in arbitrary units. The experimental PLIF image shows evidence of turbulent structures on the edges of the jet's shear layer. The scale and distribution of turbulent structures might be misleading, however. Because the three-dimensional jet was created by combining a series of uncorrelated single-shot PLIF images, large variations in spatial structure between neighboring slices can result in an artificially corrugated surface. Through smoothing of the experimental data in Tecplot, it is possible to filter the artificial small-scale structures but retain real large-scale fluid dynamic structures. Figure $6 \mathrm{c}$ shows the data set after it has been filtered with 400 passes of Tecplot's smoothing algorithm. The default relaxation factor of 0.5 was specified in the smoothing algorithm.

a)

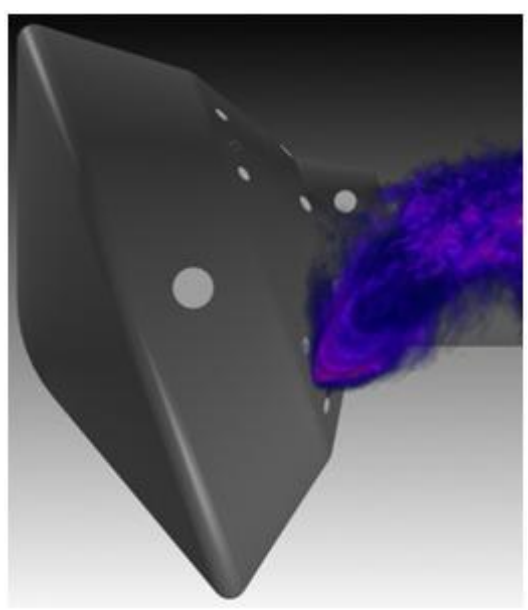

b)

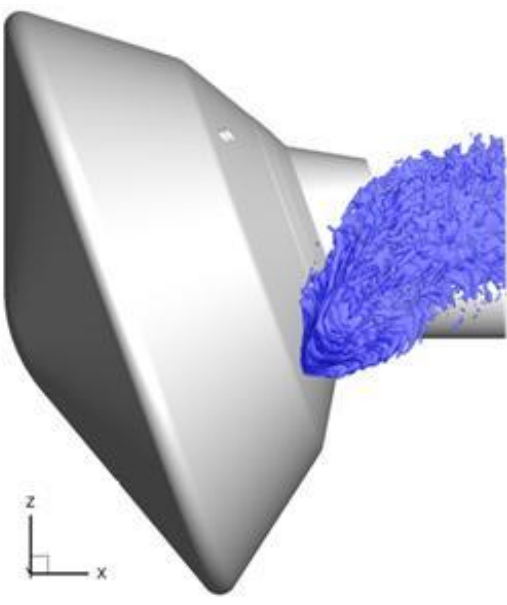

c)

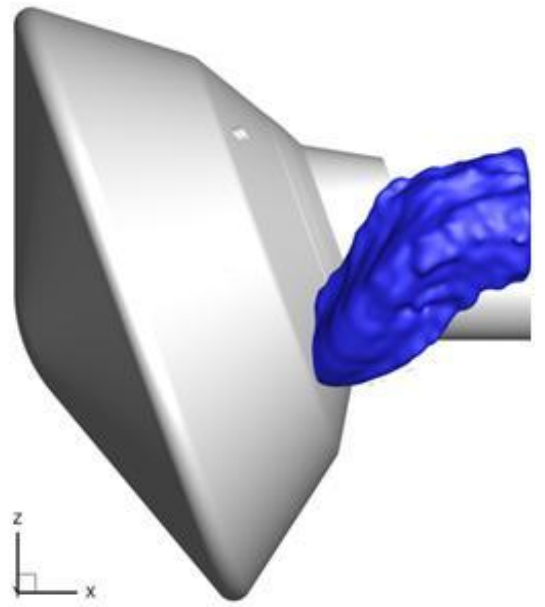

Figure 6. Visualization of RCS Jet B2. a) ViDI visualization reproduced from Ref. 4; b) Tecplot visualization of PLIF signal iso-contour surface for $S_{f}=20$ (un-smoothed); c) Tecplot visualization of PLIF signal isocontour surface for $S_{f}=10$ (smoothed).

Figure 7 compares a computed RCS jet structure with both smoothed and un-smoothed experimental PLIF isosurfaces. The computed PLIF iso-surface is based on an empirical CFI model developed by Inman for an underexpanded jet. ${ }^{19}$ The PLIF signal, $S_{f}$, is calculated from the following expression:

$$
S_{f} \propto \chi_{N O}\left\{(-0.0018) T^{-2.12} P^{2}+(46.0) T^{-1.9} P\right\}
$$

In the expression, $\chi_{\mathrm{NO}}$ is the NO mole fraction, $T$ is the gas temperature in Kelvin, and $P$ is the gas pressure in Pascals. Since pure $\mathrm{N}_{2}$ was used as the RCS jet fluid in the CFD simulations, a mole fraction ratio of $\chi_{\mathrm{NO}} / \chi_{\mathrm{N} 2}=0.05$ was assumed. The empirical model is valid over the temperature range of $100 \mathrm{~K}$ to $500 \mathrm{~K}$ and a pressure range of 2 $\mathrm{Pa}$ to $30 \mathrm{kPa}$. Rapid repopulation of the ground state, negligible saturation of the fluorescence transitions, a tripleGaussian spectral overlap integral representation, and negligible quenching from $\mathrm{O}_{2}$ are assumed. Full details of the model can be found in Refs. 19 and 20. The model was also used to perform a similar comparison between experiment and CFD where RCS jet interaction of the Crew Exploration Vehicle was investigated. ${ }^{21}$ 

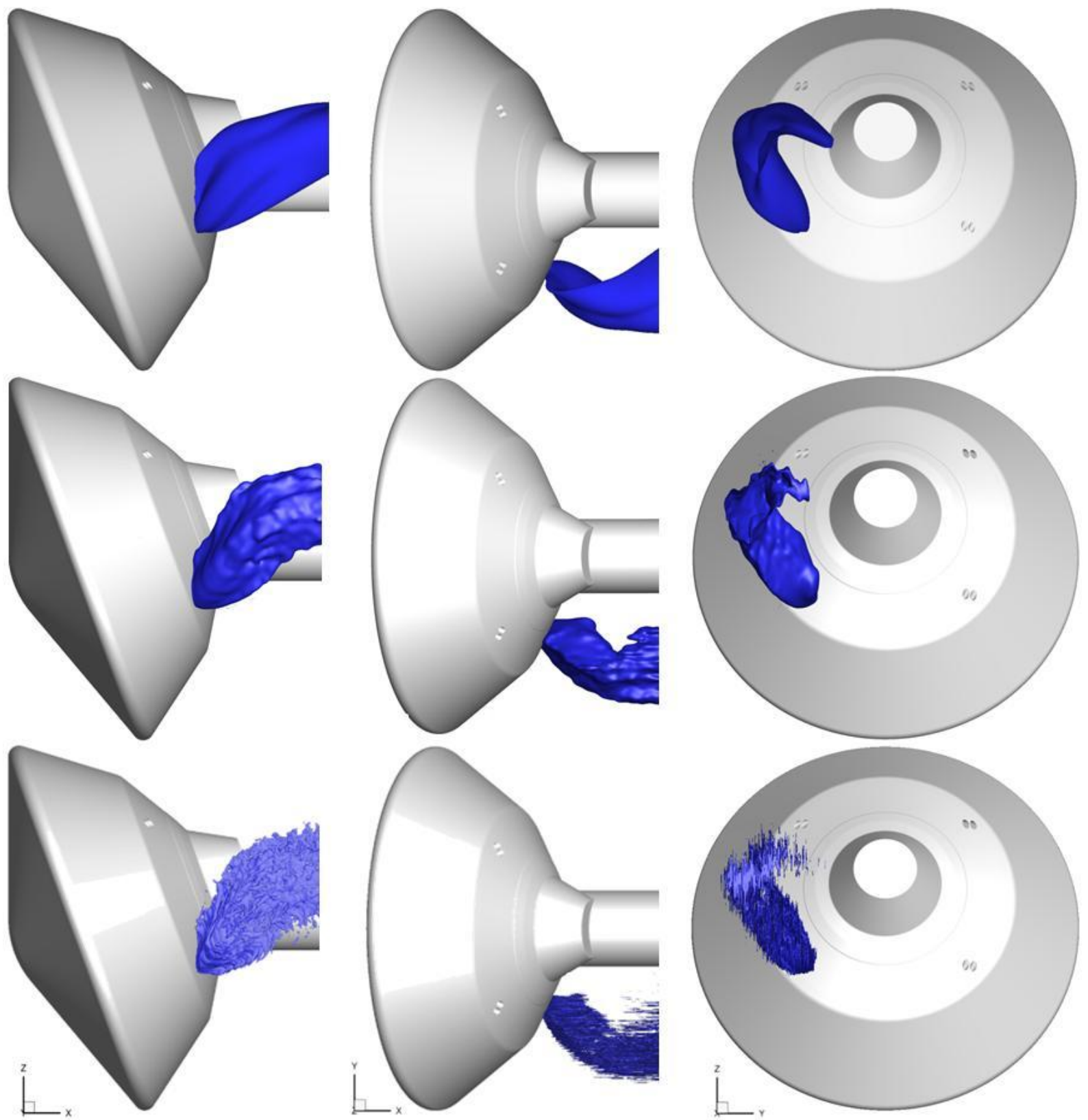

Figure 7. Comparison of computed $S_{f}=0.005$ (first row), smoothed experimental $S_{f}=10$ (second row) and unsmoothed experimental $S_{f}=20$ (third row) RCS Jet iso-contour surfaces. Note that the sting, present in the experiments and computations, has been removed from the $\boldsymbol{y}-z$ view for clarity.

The computed jet structure in Fig. 7 corresponds to an iso-contour value of $S_{f}=0.005$. For the smoothed and unsmoothed experimental data, the structure corresponds to a PLIF signal iso-value of $S_{f}=10$ and $S_{f}=20$, respectively. The iso-value was specified for each case so that the jet structure near the nozzle exit was approximately the same size. Qualitatively, the shapes and trajectories of the jets predicted by OVERFLOW agree well with the experiment in each of the three views. Small scale turbulence structure cannot be visualized from the RANS-type numerical simulations as the entire turbulence spectrum was modeled. Even with data smoothing, the lack of measurements at some planes caused relatively large distortions in the overall shape (see $y-z$ view). Although the jet nozzles are conical, both the experiment and computations show larger growth of the jet in the $z$ - direction compared to the $y$ direction. Because the individual RCS jets are closely spaced, it is likely that the jet-jet interactions promote greater expansion in the $z$ - direction compared to the $y$ - direction. The initial jet trajectory direction is away from the sting (- 
y) but then reverses direction back towards the sting further downstream. The redirection of the jet towards the sting occurs from the interaction of the jet with the high-speed outer flow.

Typically, visualization of the jet structure from CFD involves plotting an iso-surface of a single flow parameter such as pressure, Mach number, or mixing fraction. For example, Fig. 8a shows an iso-surface of $\chi_{\mathrm{NO}}=0.038$ (mole fraction of $\mathrm{NO}$ is a parameter equivalent to jet fluid mixing fraction for this set of computations). By combining multiple parameters using Eq. (1), CFI creates an alternative visualization of the jet structure by generating isosurfaces of computed fluorescence intensity. Figure $8 \mathrm{~b}$ shows the influence of the CFI model on the visualized shape of the jet structure with a selected iso-surface of $S_{f}=0.005$. The $\chi_{\mathrm{NO}}$ iso-surface value was selected to match the jetstructure and size of the CFI model iso-surface near the nozzle exit. Note that although the shape and trajectory of the jet structures are similar, the CFI model predicts an iso-surface that persists further downstream of the capsule.

a)

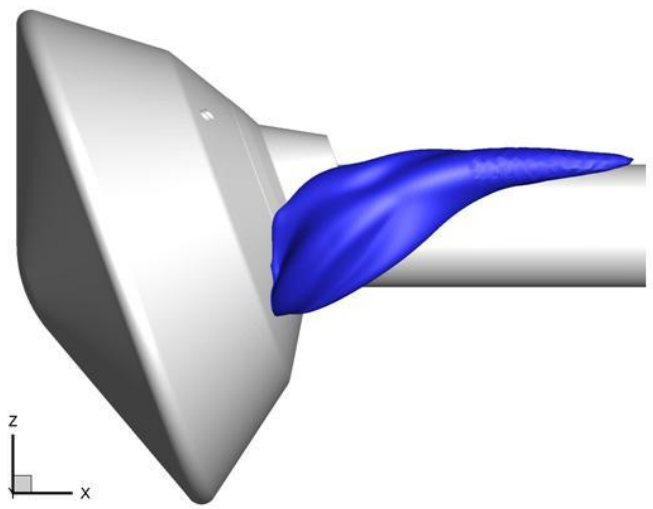

b)

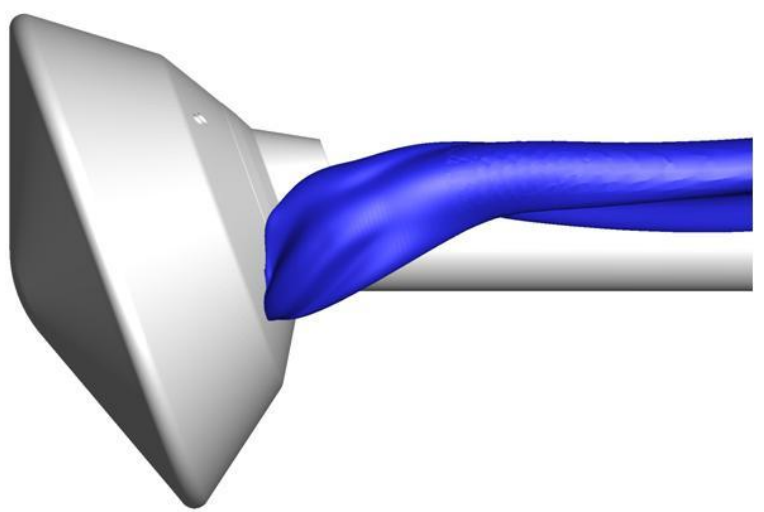

Figure 8. Effect on RCS jet structure of plotting a CFD flow parameter versus using a CFI model: a) Isosurface of $\chi_{N O}=0.038$; b) Iso-surface of $S_{f}=0.005$.

Upon further analysis of the simulation solution, it was found that shock compression of the RCS jet in the capsule wake results in an increase in gas density and ultimately an increase in PLIF signal intensity. The effects of gas density are indirectly accounted for in Eq. (1) from the combination of pressure and temperature terms. Figure 9 shows the interaction of the RCS jet with the outer-flow, interaction with the first oblique shock wave that originates from the sting, and interaction with the second oblique shock that originates from the wedge further downstream on the sting. As shown in the figure, each of these interactions results in an increase in PLIF signal for a constant NO mole fraction. Prior to the shock wave interactions the jet structure appears to be mostly dominated by the NO mole fraction distribution as it forms in the subsonic wake near the capsule surface (see Fig. 8).

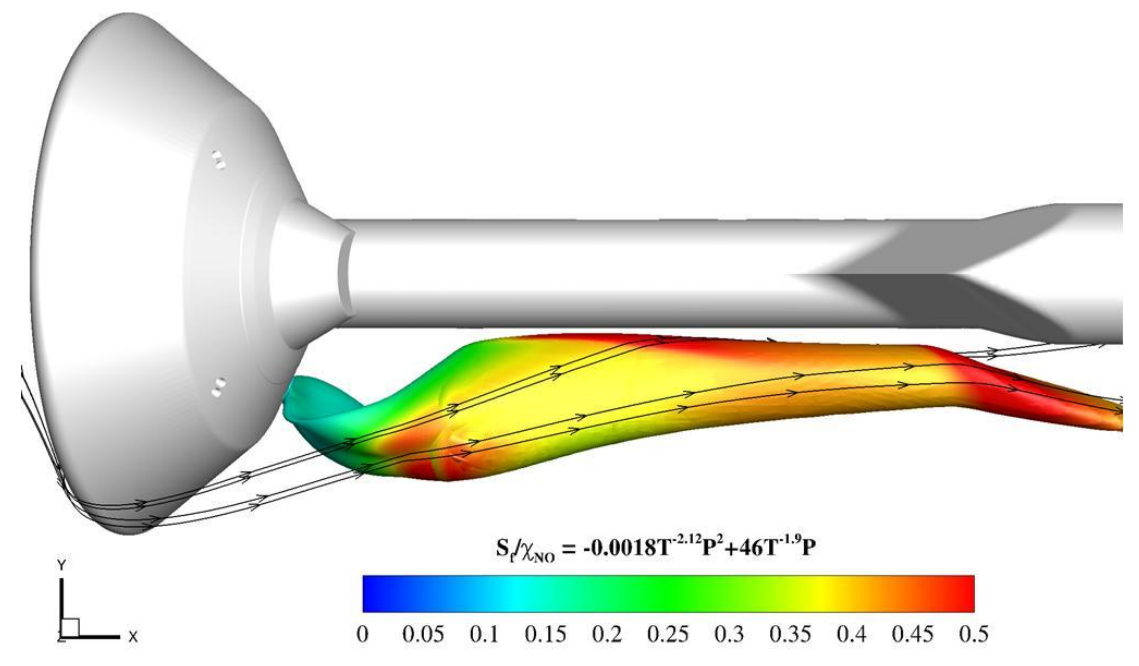

Figure 9. CFI result showing dependence of fluorescence signal on factors other than mole fraction of NO, including the effect of shock wave interaction. Iso-contour of $S_{\mathrm{f}}=0.005$ colored by contours of $S_{f} / \chi_{\mathrm{NO}}$. 
Figure 10 shows the jet trajectory for both the simulation and experiment based on post-processing of the respective PLIF signals. Using a Tecplot script, each point is calculated from signal-weighted average over a $y-z$ plane at a given $x$ position:

$$
y_{c}=\frac{\sum\left(\left(S_{f}\right)_{i} y_{i}\right)}{\sum\left(S_{f}\right)_{i}}, z_{c}=\frac{\sum\left(\left(S_{f}\right)_{i} z_{i}\right)}{\sum\left(S_{f}\right)_{i}}
$$

In Eq. (2), $y_{c}$ and $z_{c}$ are the $y$ and $z$ coordinates corresponding to the center of the jet. The trajectory is constructed as the calculation is performed at subsequent downstream $y$ - $z$ planes. A similar technique was used to calculate the two-dimensional trajectory from two dimensional PLIF images of the RCS jets for an Apollo-geometry capsule. ${ }^{22}$ Figure 11 shows a direct comparison between the $x-y$ and $x-z$ jet trajectories based on the experimental and computed flow visualization data. In the experimental flow visualization data, a threshold of $S_{f}=20$ was applied to Eq. (1) to remove artificial effects of noise on the calculation far away from the jet center.
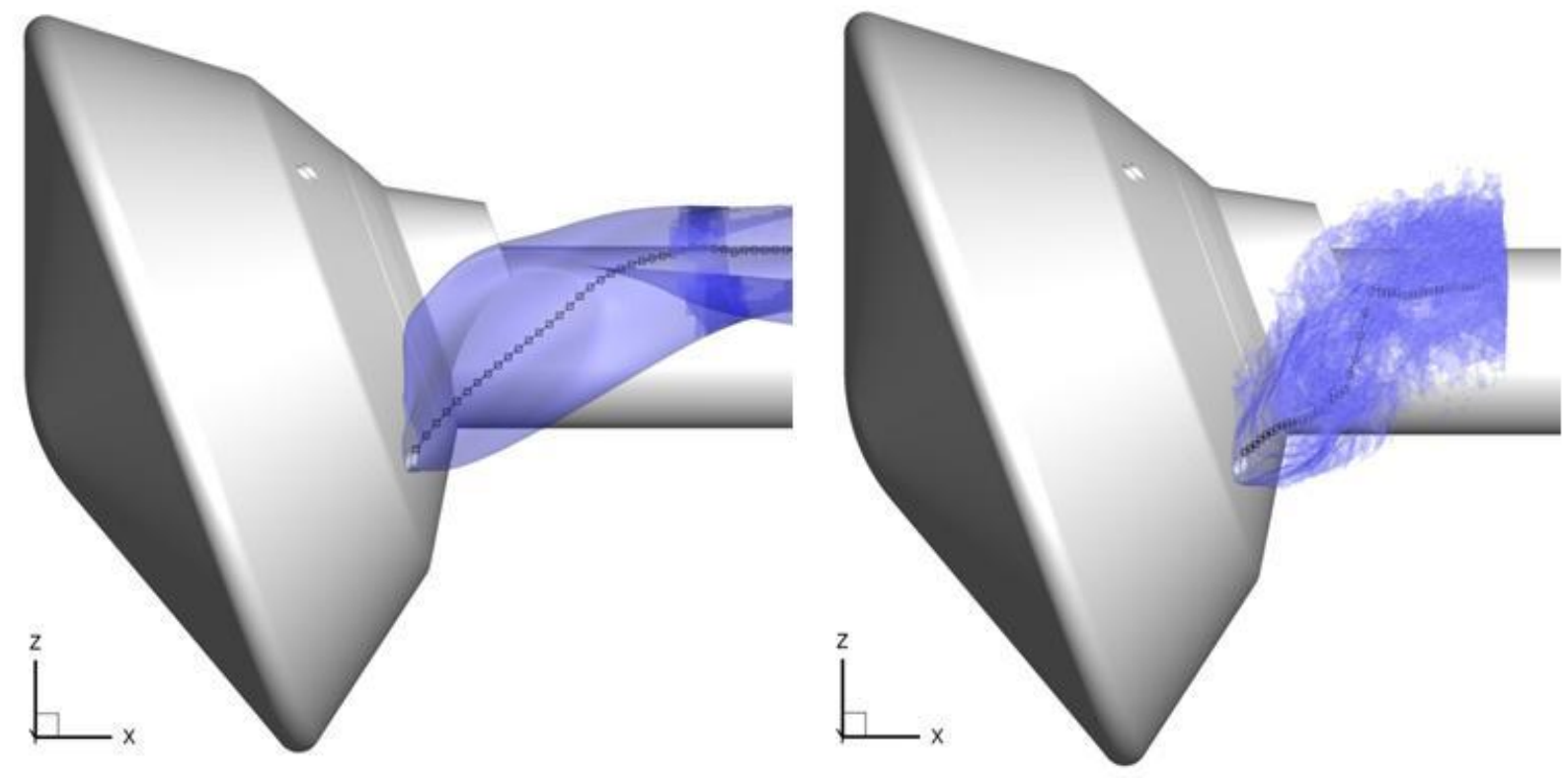

Figure 10. RCS jet trajectory based on computed (left) and experimental (right) signal iso-contour surfaces.

Also shown in Fig. 11 is the effect of applying a threshold to the calculation of the trajectory. Results indicate that in the simulation, the trajectory near the nozzle exit is unaffected by the threshold level. There is, however, some sensitivity of the calculation to threshold levels further downstream. As the NO diffuses away from the jet core and peak concentration levels decrease, lower thresholds are required to accurately predict the jet trajectory. Overall, the agreement between the simulation and experiment is marginal. Errors are most likely caused by the threshold method applied to the experimental data and some inaccuracy of the empirical PLIF model. Lower thresholds could not be applied to the experimental data since the jet is not centered on the measurement volume and increased noise levels near the sting bias the trajectory in the $+y$ direction. Better signal-to-noise measurements in the experiment would improve the accuracy of the trajectory calculation. 

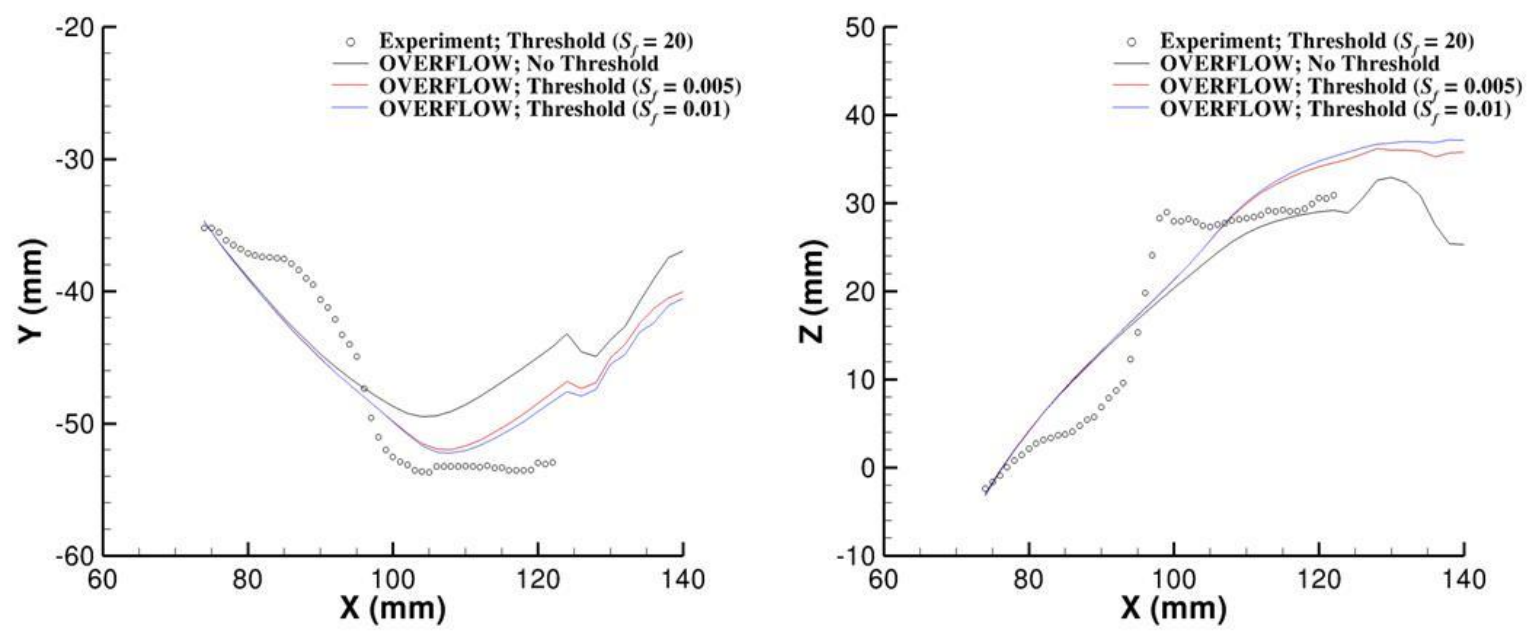

Figure 11. Comparison of the computed and experimental jet trajectory shown in Fig. 10. The effect of threshold levels on the predicted trajectory is shown.

\section{Velocity Measurement Comparison}

NO PLIF molecular tagging velocimetry was used to characterize the streamwise velocity field in the wake flow with and without the RCS B2 jet pair activated. When the RCS jet pair was on, NO was seeded both into the RCS jets and from the junction between the model and sting. When the RCS jet was off, NO was seeded only from the junction between the model and sting. For each RCS jet operational mode (jet on or jet off), the laser sheet traversed a set of six equally spaced spanwise distances, stopping at each location for approximately 2 seconds to allow for 20 images to be captured at each location for statistical analysis. Through post-processing, the quantitative distribution of streamwise velocity along each of the MTV lines was measured. A contour map of streamwise velocity was created through the interpolation of each of the MTV lines onto a two dimensional plane using Tecplot. A sample of one of the contour maps of streamwise velocity $\left(V_{x}\right)$ without the RCS jets activated is shown in Fig. 12 (right) along with the corresponding prediction from OVERFLOW (left). In this figure, the laser sheet is positioned at $y=-39$ $\mathrm{mm}$, which is the spanwise position measured from the model centerline. This spanwise position is roughly one nozzle diameter away from the activated RCS jet pair.

Since the RCS jet is not activated, the magnitude of streamwise velocity in the separated wake region is very low compared to the speed of the supersonic outerflow. Furthermore, NO gas seeded through the sting is unable to diffuse through the shear layer into the high-speed outerflow. As a result, the field of view of the measurement is limited to the size of the wake. With low signal-to-noise levels associated with the wake-seeding technique and a limited number of data points obtained during each run, the measurement error is of the same order of magnitude as the measurement itself. Although the majority of velocity measurements were low in magnitude, higher velocity magnitudes were measured in the top shear layer. Unsteadiness in the wake region, which is confirmed from the visualization images in Fig. 5, will cause an error in the position of the shear layer. MTV measurements will overpredict the position of the shear layer since only high signal (low magnitude velocity) measurements will be included in the average measurement. NO velocity measurements in the shear layer and in the outerflow could be obtained if the NO gas were seeded upstream from the capsule's forebody. The problem, unfortunately, with forebody seeding is that there is a risk that the seeded gas will inadvertently perturb the attached boundary layer flow. Since the velocity distribution in the wake is relatively uniform, only the overall velocity magnitude and shear layer location can be compared to the experiment. Further comparisons between the jet-off CFD and experimental data in four additional spanwise planes are available in the appendix. 


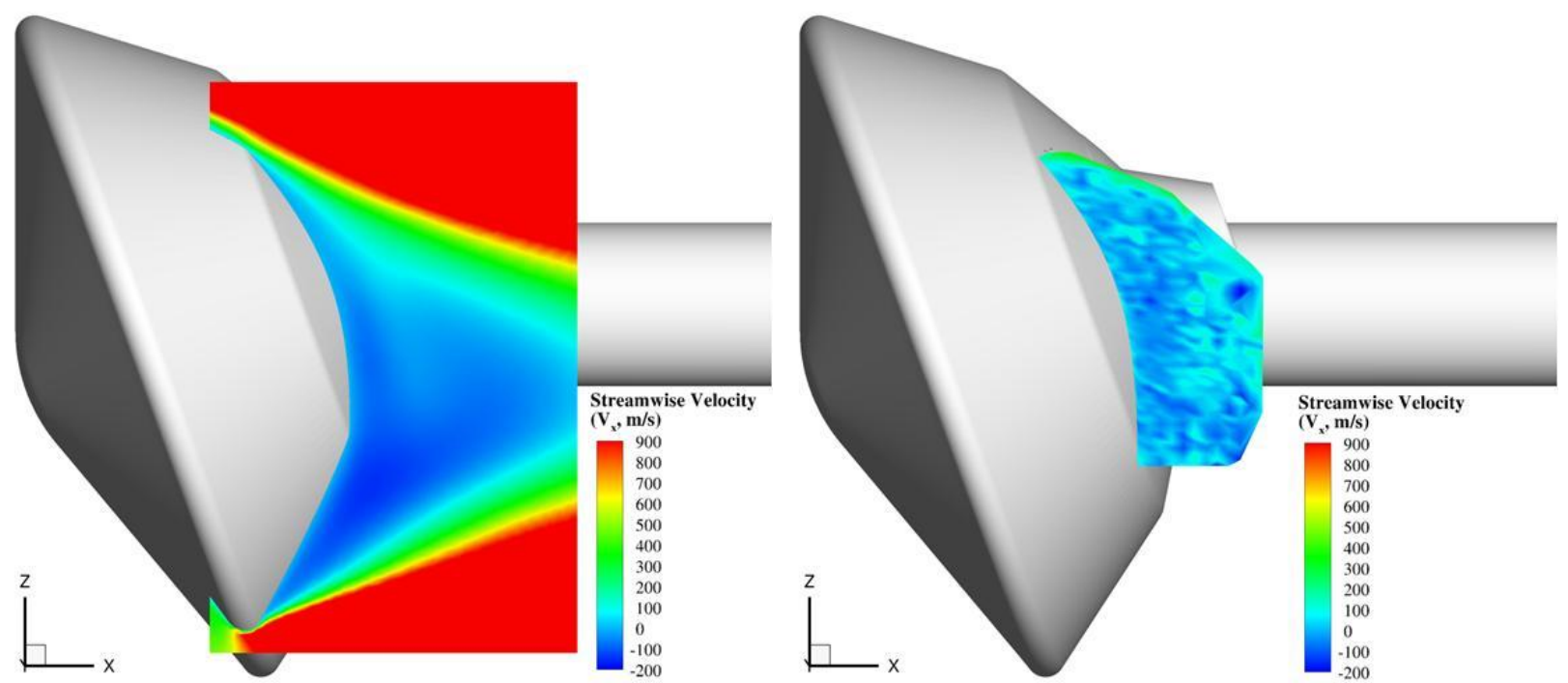

Figure 12. Contour map of CFD predicted (left) and experimentally measured (right) streamwise velocity $\left(V_{x}\right)$. Laser sheet positioned at $y=-\mathbf{- 3 9} \mathbf{~ m m}$ from the model centerline. RCS jet is deactivated.

Figure 13 shows a contour map of the computed (left) and experimentally measured (right) streamwise velocity at the same spanwise position as that shown in Fig. 12 but with the RCS jets activated. Unlike the jet-off case, large magnitudes of streamwise velocity are measured in the wake. Because the RCS jets are internally seeded with NO gas, good signal-to-noise levels are achieved within the jet. As a result, a comparison between the computed and experimental RCS jet structure can be made. The simulation under-predicts the streamwise velocity within the jet core by about $10 \%$ but has good agreement on the overall shape and size of the jet. High magnitudes of velocity on the model surface are not expected and are an artifact of the laser scatter in the experiment.
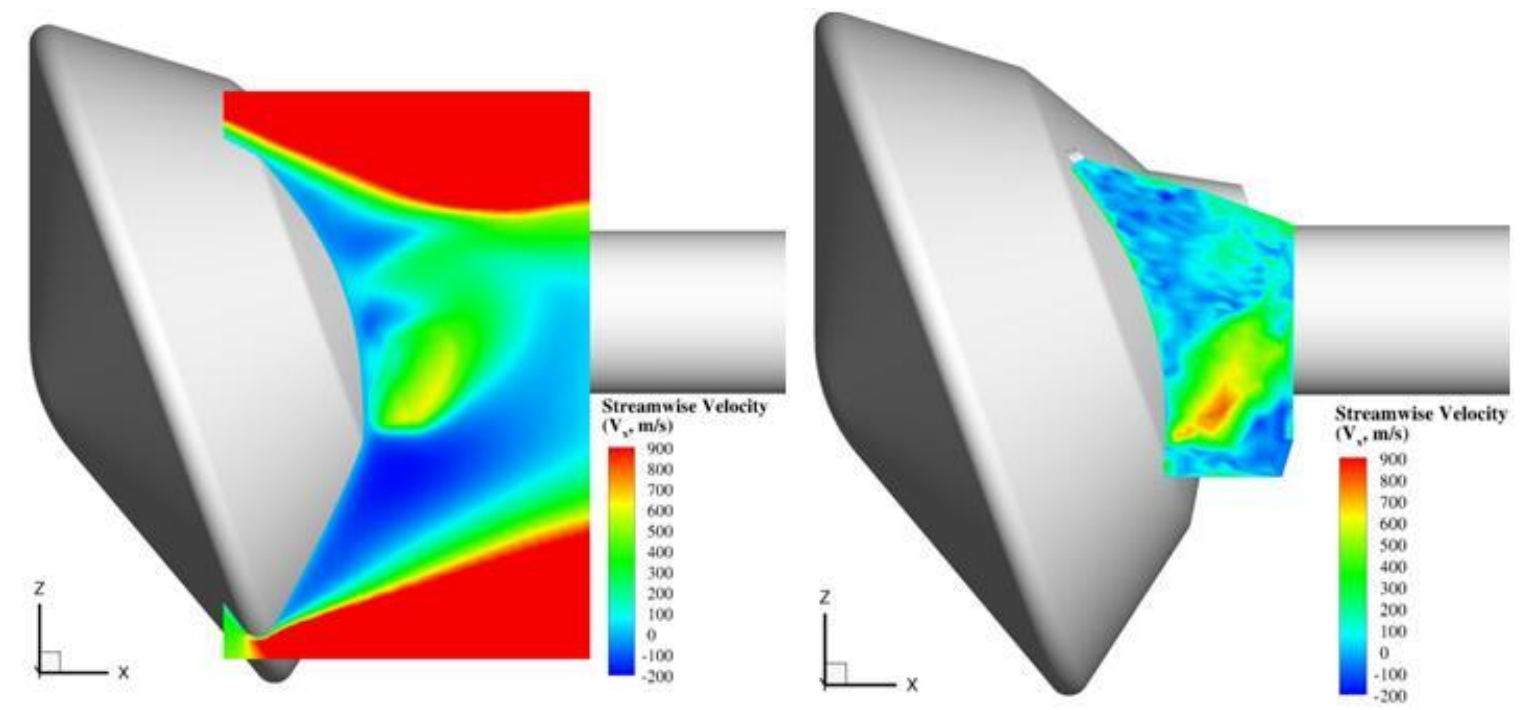

Figure 13. Contour map of CFD predicted (left) and experimentally measured (right) streamwise velocity $\left(V_{x}\right)$. Laser sheet positioned at $y=\mathbf{- 3 9} \mathbf{~ m m}$ from the model centerline. RCS jet is activated.

The simulation does predict a region of high-velocity gas above the main jet structure, which although subtle, also appears in the experimental data. Further analysis of the CFD simulation reveals that this high-velocity gas region corresponds to the core of the counter-clockwise rotating vortex described in the Wake Flow Visualization section. It is possible that the interaction between the low-pressure vortex core and the RCS jet leads to unwanted changes in the surface pressure distribution with regards to control authority. This interaction is very sensitive to the model's 
AoA as both the strength and location of the counter rotating vortex are affected. Further two-dimensional comparisons between the jet-on CFD and experimental data in three additional spanwise planes are available in the appendix.

Figure 14 shows the same computed velocity field as the left figure in Fig. 13 but with dashed lines indicating the locations of the individual measured velocity profiles. The individually measured streamwise velocity profiles along these lines corresponding to the jet-off and jet-on cases are shown in Fig. 15 and 16, respectively, along with the CFD predictions. The width of the symbols in Figs. 15 and 16 indicates the 95\% confidence uncertainty bounds of the measured mean velocity at that location. For the most part, the predictions are within the uncertainty bands of the experimentally measured velocities.

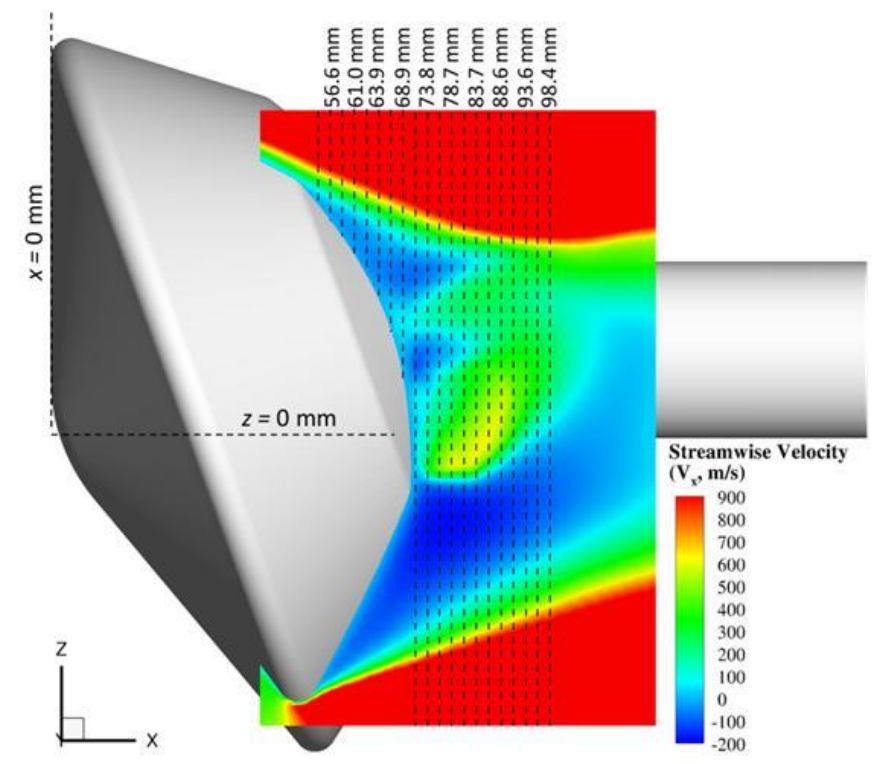

Figure 14. Contour map of computed streamwise velocity $\left(V_{x}\right)$ indicating the positions of the measured velocity profiles.

Confirming what was observed qualitatively in Fig. 12, comparisons between computed and experimental velocity profiles show that the simulations under-predict the height of the shear layer location. Placement of the experimental MTV data relative to the model was based on the location in the images of laser scatter off the surface of the model. As described above it is likely that there is a biased error in the location of the shear layer because of the unsteadiness in the wake structure and the systematic rejection of low-signal measurements when the outerflow penetrates the region of interest. 

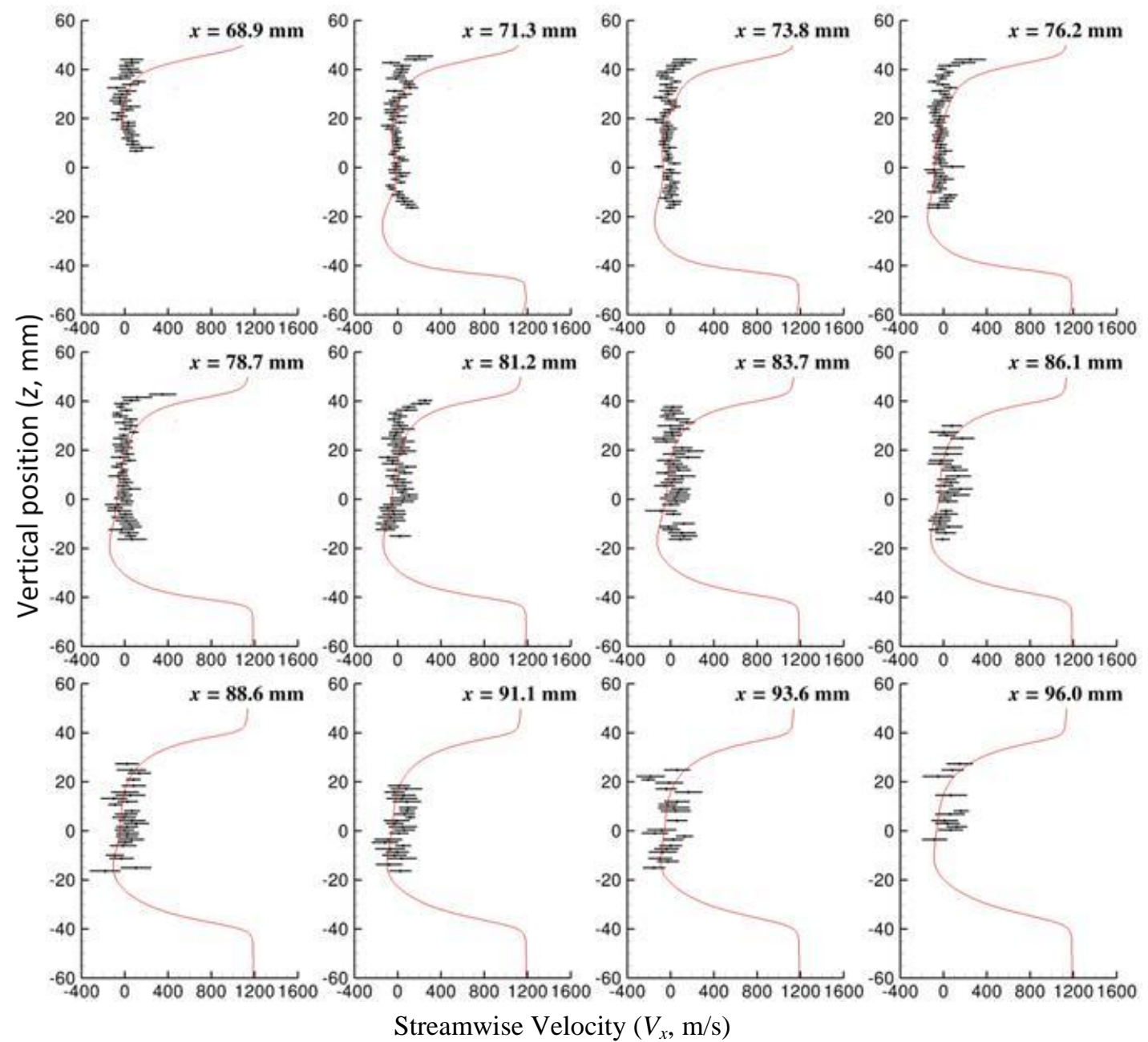

Figure 15. Vertical $(z)$ distribution of streamwise velocity $\left(V_{x}\right)$ for the conditions of Fig. 12 at $y=-39 \mathrm{~mm}$. RCS jet is deactivated. Width of bars indicates uncertainty in the mean values.

As expected the measurement error within the RCS jet core is relatively low because of the high signal-to-noise ratio in the PLIF images. There is good agreement between the predicted and experimental vertical location corresponding to peak velocity. Confidence in the vertical placement of the MTV data indicates that the poor prediction in the shear layer location is likely due to a bias error in the MTV measurement. It is also possible that the prediction accuracy of the separation location is limited by the RANS-type turbulence modeling. RANS-type turbulence models traditionally have difficulty predicting flows with streamline curvature and flow separation. ${ }^{9}$ Further analysis is needed to determine the discrepancy in the shear layer location. Another discrepancy between the simulation and experiment is the prediction of the peak magnitude of velocity. At each streamwise location, the simulations under-predict the magnitude of peak velocity. Although additional comparisons in the appendix indicate that the peak velocity is sensitive to the spanwise location, the peak velocity extracted from the MTV measurements is always larger than the predicted peak values. The under-prediction of peak streamwise velocity magnitudes can be partially attributed to an error in the predicted jet orientation. For example, the initial $x-z$ and $x-y$ trajectory measurements $(75 \mathrm{~mm}<x<95 \mathrm{~mm})$ shown in Fig. 11 indicate that the predicted ratios of streamwise-to-vertical $\left(V_{z} / V_{x}\right)$ and streamwise-to-spanwise $\left(V_{y} / V_{x}\right)$ velocity components are lower than the ratios calculated from the experiment. As a result, the predicted total velocity magnitude could be accurate but include errors in the predicted velocity component ratios (i.e. $V_{z} / V_{x}$ and $V_{y} / V_{x}$ ). The observation in the trajectory described above occurs over a streamwise range $(75 \mathrm{~mm}<x<95 \mathrm{~mm})$ that is coincident with measurements shown in Fig. 16 and Figs. A10-A11. It is also possible that an NO LIF quenching gradient exists in the streamwise direction and caused a bias error in the calculation of the velocity magnitudes from the experimental MTV data. ${ }^{16}$ Furthermore, the MTV velocity 
calculations might be sensitive to the maximum signal intensity threshold criteria used to reject large signals in the first camera gate that artificially deplete signals in the second camera gate. Depletion of signals in the second camera gate will bias the velocity measurement to higher values.
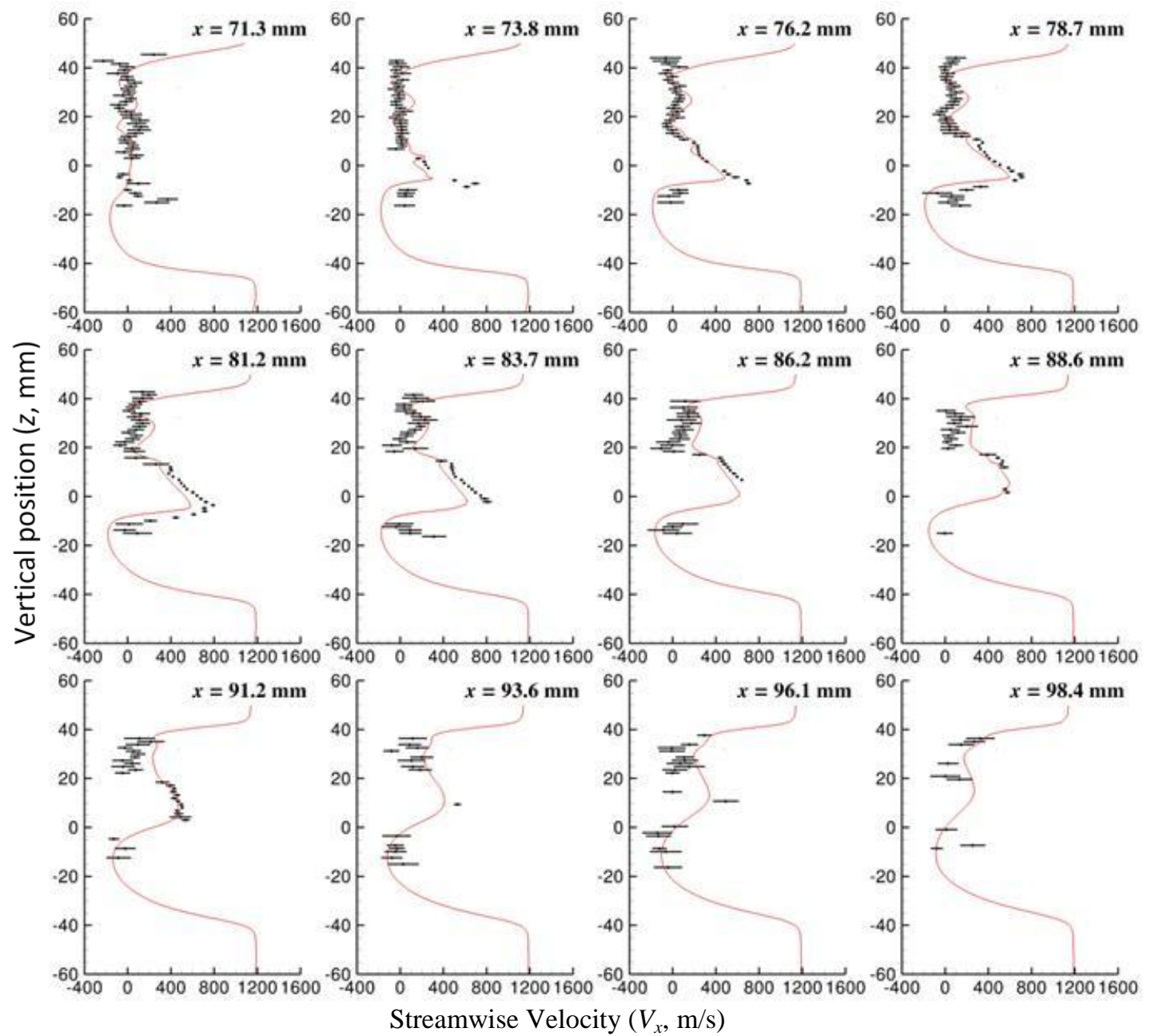

Figure 16. Vertical $(z)$ distribution of streamwise velocity $\left(V_{\mathrm{x}}\right)$ for the conditions of Fig. 13 at $y=-39 \mathrm{~mm}$. RCS jet is activated. Width of bars indicates uncertainty in the mean values.

\section{Discussion and Future Work}

The numerical simulation solutions have identified important aerodynamic structures in the wake of the MSL vehicle that are not currently captured by the existing PLIF dataset. A more in depth study of the wake should include measurements further downstream to capture the shape of the entire separated region. Of special interest is the presence of a counter-rotating vortex pair that entrains seeded NO from the sting and transports it far downstream of the capsule. Future PLIF experiments that can extract the vorticity from these trailing vortices could be used to predict the circulation generated in the near-wake flow field. The circulation could be related to the aerodynamic forces acting on the vehicle through a process analogous to Prandtl's lifting line theory. The MTV technique used with cameras re-oriented in the streamwise direction might be used to capture the $x$-component of vorticity. A two-component (cross-pattern) MTV velocity measurement would capture vorticity in the separation regions along the centerline. NO seeding from the forebody will also enable better visualization of the shear layer and make the MTV measurements less susceptible to bias errors. The current MSL model has ports on the forebody to enable this type of seeding. In future tests, the MTV velocity measurements would be improved if the NO wake seeding flow rate were increased to improve the signal-to-noise ratio. In addition, improvements to the MTV technique would be made if the NO concentration in the RCS jets were decreased to avoid saturation of the camera's 
CCD. Performing detailed wake and RCS jet PLIF measurements at different model AoAs would strengthen the numerical model validation. Further development of the CFI modeling is required to produce more accurate predictions of the jet structure for comparison to the experimental PLIF images.

\section{Conclusions}

A validation study of the OVERFLOW code has been performed through qualitative and quantitative comparisons of the predicted MSL wake and RCS jet flow field to an experimental PLIF dataset. Overall there is good agreement between the experiment and CFD with regards to the size and shape of the separated recirculation zones in the capsule wake. A counter-rotating vortex pair was identified from the numerical simulations, the effect of which was not deduced from the experiments alone. The simulations predict entrainment of NO seeded from the sting and RCS jets into the vortex cores, allowing for potential experimental PLIF measurements in the far field in a future experiment. A CFI model was applied to the CFD solution to make comparisons between the predicted and experimental RCS jet structure. The effect of smoothing on the experimental data was investigated and the processed images were compared to CFD from several different views. The CFD solution was used to investigate the limits of the CFI model. It was found that near the capsule surface the jet structure based on the PLIF signal is dominated by mole fraction of NO. Further downstream the interaction of the jet with a series of shock waves influences the signal levels and increases the perceived jet size and length compared to structures based on isosurfaces of NO mole fraction. For the first time, a three-dimensional jet trajectory was extracted from both the experimental and CFI images and compared. Agreement between the experiment and CFD based on the jet trajectory was marginal. Experimental NO PLIF MTV measurements were compared both qualitatively and qualitatively for jet-on and jet-off cases at several spanwise positions in the capsule wake. There was good agreement in the regions with high signal-to-noise ratio, such as in the RCS jets. Low signal-to-noise levels in the experimental PLIF data away from the RCS jets restricted the comparison for the jet-off case. Distributions of highvelocity gas predicted by CFD and confirmed in the MTV measurements were found to coincide with the core of the streamwise trailing vortex.

\section{Acknowledgements}

This work was supported by the NASA Engineering and Safety Center (NESC) located at NASA Langley Research Center, Hampton Virginia, under the supervision of David Schuster. Support was also provided by the NASA Fundamental Aeronautics Program, Hypersonics Project. We wish to acknowledge the expert assistance of the 31-Inch Mach 10 Air Tunnel staff and the team of engineers that assisted with the MSL wind tunnel model development and wind tunnel test at NASA Langley. These include Mark Schoenenberger, John Paulson, John Van Norman, Matthew Rhode and Vince Le Boffe. Dr. Johansen was supported by the Natural Sciences and Engineering Research Council of Canada (NSERC).

\section{References}

1 A.A. Dyakonov, M. Schoenenberger, W.I. Scallion, J.W. Van Norman, L.A. Novak, C. Y. Tang, "Aerodynamic Interference Due to MSL Reaction Control System," $41^{\text {st }}$ AIAA Thermophysics Conference, San Antonio, TX, AIAA Paper 2009-3915, June. 2009.

${ }^{2}$ K.T. Edquist, A.D. Dyakonov, M.J. Wright, and C.Y. Tang, "Aerothermodynamic Design of the Mars Science Laboratory Backshell and Parachute Cone," AIAA Paper 2009-4078 $41^{\text {st }}$ AIAA Thermophysics Conference, San Antonio, TX, June 22-25, 2009.

${ }^{3}$ M. Schoenenberger, A. Dyakonov, P. Buning, W. Scallion, and J.Van Norman, "Aerodynamic Challenges for the Mars Science Laboratory Entry, Descent and Landing," AIAA Paper 2009-3914 41 ${ }^{\text {st }}$ AIAA Thermophysics Conference, San Antonio, TX, June 22-25, 2009.

${ }^{4}$ C.T. Johansen, P.M. Danehy, S.W. Ashcraft, B. Bathel, J.A. Inman, and S.B. Jones, "PLIF study of Mars Science Laboratory capsule reaction control system jets" 41st AIAA Fluid Dynamics Conference and Exhibit, Honolulu Hawaii, 27 - 30 Jun 2011.

${ }^{5}$ K.T. Edquist, A.A. Dyakonov, M.J. Wright, and C.Y. Yang, "Aerothermodynamic Environments Definition for the Mars Science Laboratory Entry Capsule," AIAA Paper 2007-1206, 45 ${ }^{\text {th }}$ AIAA Aerospace Sciences Meeting and Exhibit, Reno, NV, January 8-11, 2007 
6 A.A. Dyakonov, K.T. Edquist, and M. Schoenenberger, "Influence of the Angle of Attack on the Aerothermodynamics of the Mars Science Laboratory," AIAA Paper 2009-3889, 36 ${ }^{\text {th }}$ AIAA Fluid Dynamics Conference and Exhibit, San Francisco, CA, June 5-8, 2006.

7 D.M. Peterson and G.V. Candler, "Detached Eddy Simulation of RCS-Aerodynamic Interaction of Mars Science Laboratory Capsule," AIAA Paper 2008-624 46 ${ }^{\text {th }}$ AIAA Aerospace Sciences Meeting and Exhibit, Reno, Nevada, Jan. 7-10, 2008.

${ }^{8}$ K.T. Edquist, A.A. Dyakanov, M.J. Wright, and C.Y. Tang, "Aerothermodynamic Design of the Mars Science Laboratory Heatshield," AIAA Paper 2009-4075, 39 th AIAA Fluid Dynamics Conference and Exhibit, San Antonio, Texas, June 22-25, 2009.

${ }^{9}$ P.R. Spalart, "Strategies for turbulence modelling and simulations," International Journal for Heat and Fluid Flow, Vol. 21, 2000, pp. 252-263.

${ }^{10}$ B. Connelly, B. Bennett, M. Smooke, and M. Long, "A paradigm shift in the interaction of experiments and computations in combustion research," Proceedings of the Combustion Institute, Vol. 32, Issue 1, 2009, pp. 879886.

11 W.M. Ruyten, W.D. Williams, F.L. Heltsley, "Computational flow imaging for planar laser-induced fluorescence applications (CFI-PLIF)," AIAA Paper 1994-2621, $18^{\text {th }}$ Aerospace Ground Testing Conference, Colorado Springs, Colorado, June 20-23, 1994.

12 P.M. Danehy, P.C. Palma, R.R. Boyce, A.F.P. Howing, "Numerical simulation of laser-induced fluorescence imaging in shock-layer flows," AIAA Journal, Vol. 37, 1999, pp. 715-722.

13 J.R. Micol, "Langley Aerothermodynamic Facilities Complex: Enhancements and Testing Capabilities," AIAA Paper 98-0147, 36th AIAA Aerospace Sciences Meeting and Exhibit, Reno, NV, Jan. 1998

${ }^{14}$ P.M. Danehy, J.A. Inman, D.W. Alderfer, G.M. Buck, B. Bathel, "Visualization of flowfield modification by RCS jets on a capsule entry vehicle", AIAA Paper 2008-1231 46th AIAA Aerospace Sciences Meeting and Exhibit, Reno, Nevada, Jan. 7-10, 2008.

${ }^{15}$ P.M. Danehy, D.W. Alderfer, J.A. Inman, K.T. Berger, G.M. Buck, and R. J. Schwartz, "Fluorescence Imaging and Streamline Visualization of Hypersonic Flow Over Rapid Prototype Wind-Tunnel Models" Proc. IMechE, Part G: J. Aerospace Engineering, Vol. 222 (G5), pp. 637-651, 2008.

16 B.F. Bathel, P.M. Danehy, J.A. Inman, S.B. Jones, C.B. Ivey, and C.P. Goyne, "Velocity Profile Measurements in Hypersonic Flows Using Sequentially Imaged Fluorescence-Based Molecular Tagging," AIAA Journal, Vol. 49, No. 9, pp. 1883-1896, September 2011..

${ }^{17}$ P. Buning, D. Jespersen, T. Pulliam, G. Klopfer, W. Chan, J. Slotnick, S. Krist, and K. Renze, "OVERFLOW User's Manual, Version 1.8s", NASA Langley Research Center, 2000.

${ }^{18}$ W.M. Chan, R.J. Gomez, S.E. Rogers, and P.G. Buning, "Best Practices in Overset Grid Generation," 32nd AIAA Fluid Dynamics Conference, St. Louis, MO, June 2002, AIAA 2002-3191.

19 J.A. Inman, "Fluorescence Imaging Study of Free and Impinging Supersonic Jets: Jet Structure and Turbulent Transition," PhD Dissertation, Dept. of Physics, The College of William and Mary, March 2007.

${ }^{20}$ J.A. Wilkes, C. Glass, P. M. Danehy and R. J. Nowak, "Fluorescence Imaging of Underexpanded Jets and Comparison with CFD” AIAA Paper 2006-910, 44th AIAA Aerospace Sciences Meeting and Exhibit, Reno, Nevada, Jan. 9-12, 2006.

${ }^{21}$ C. B. Ivey, P. M. Danehy, B. F. Bathel, A. A. Dyakonov, J. A. Inman, and S. B. Jones "Comparison of PLIF and CFD Results for the Orion CEV RCS Jets" AIAA Paper 2011-713 49th AIAA Aerospace Sciences Meeting, Fluid Dynamics TC, Orlando Florida, January 2011.

${ }^{22}$ P.M. Danehy, J.A. Inman, G. Brauckmann, D.W. Alderfer, S.B. Jones, and D. Patry, "Visualization of a Capsule Entry Vehicle Reaction-Control System Thruster," AIAA Journal of Spacecraft and Rockets, Vol. 46., no. 1, 2009, pp. 93-102. 


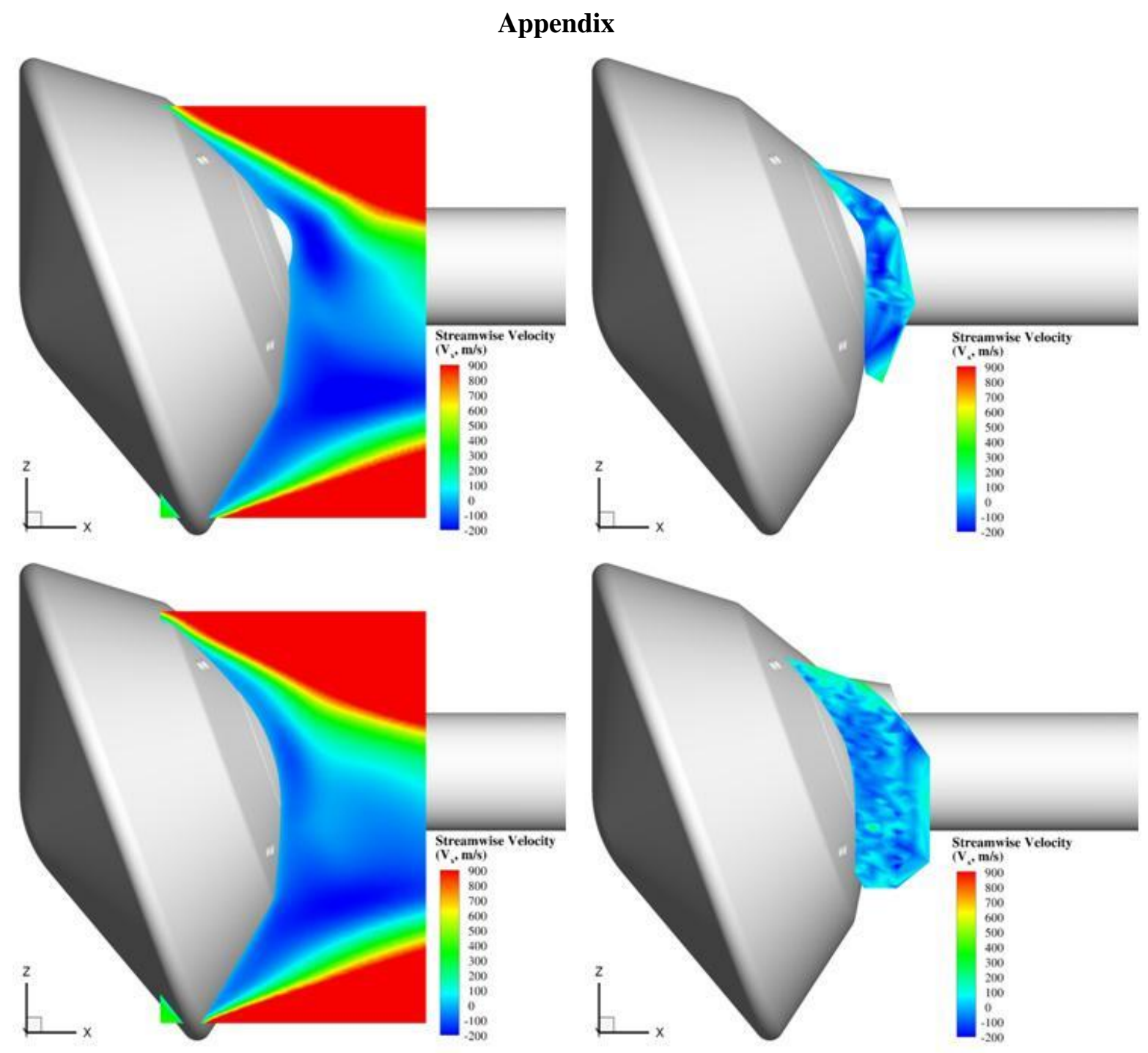

Figure A1. Contour map of CFD predicted (left) and experimentally measured (right) streamwise velocity $\left(V_{x}\right)$. Laser sheet positioned at $y=-19 \mathrm{~mm}\left(1^{\text {st }}\right.$ row $)$ and $-26 \mathrm{~mm}\left(2^{\text {nd }}\right.$ row $)$ from the model centerline. RCS jet is deactivated. 


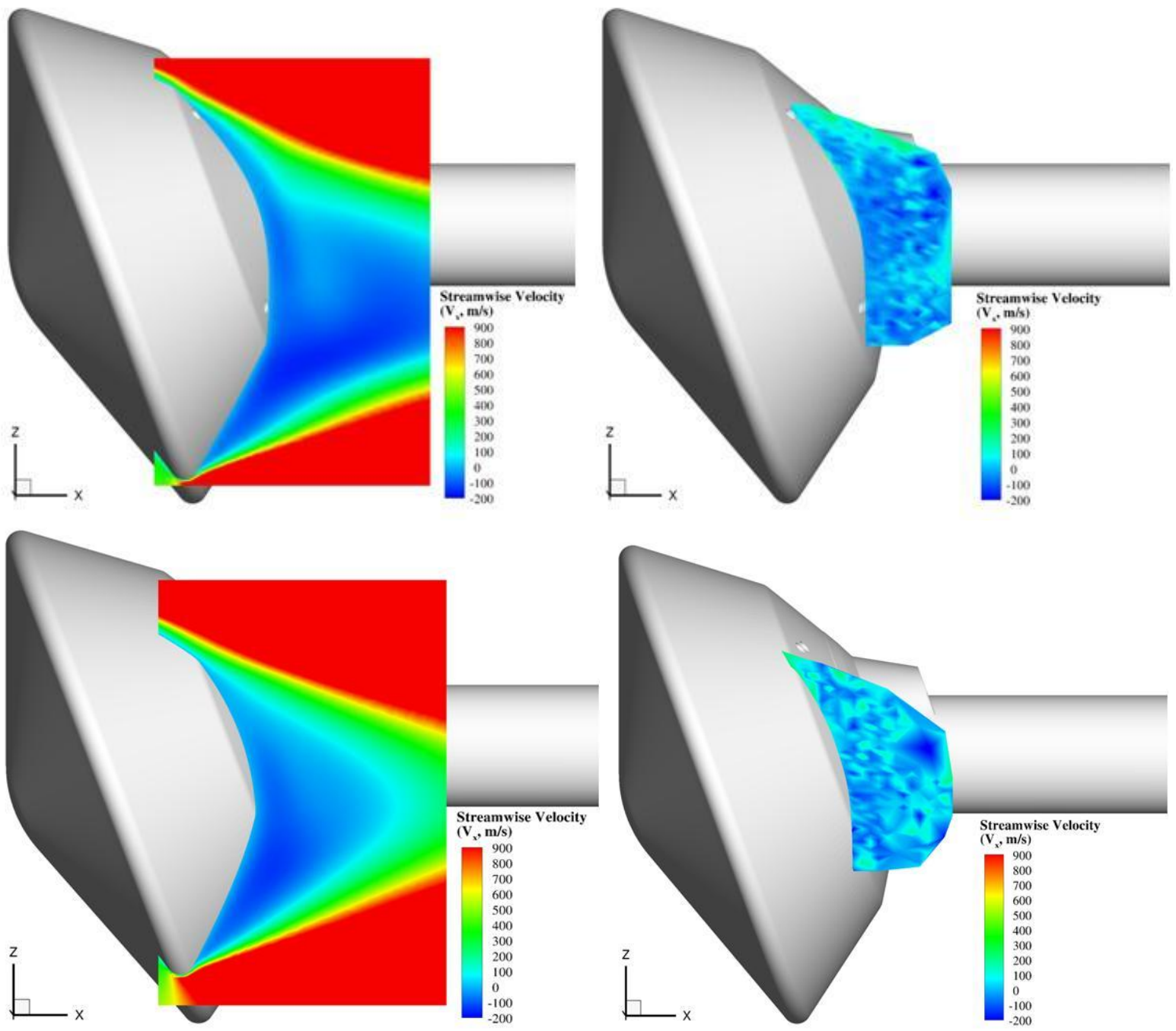

Figure A2. Contour map of CFD predicted (left) and experimentally measured (right) streamwise velocity $\left(V_{x}\right)$. Laser sheet positioned at $y=-32.5 \mathrm{~mm}\left(1^{\text {st }}\right.$ row $)$ and $-45 \mathrm{~mm}\left(2^{\text {nd }}\right.$ row $)$ from the model centerline. RCS jet is deactivated. 

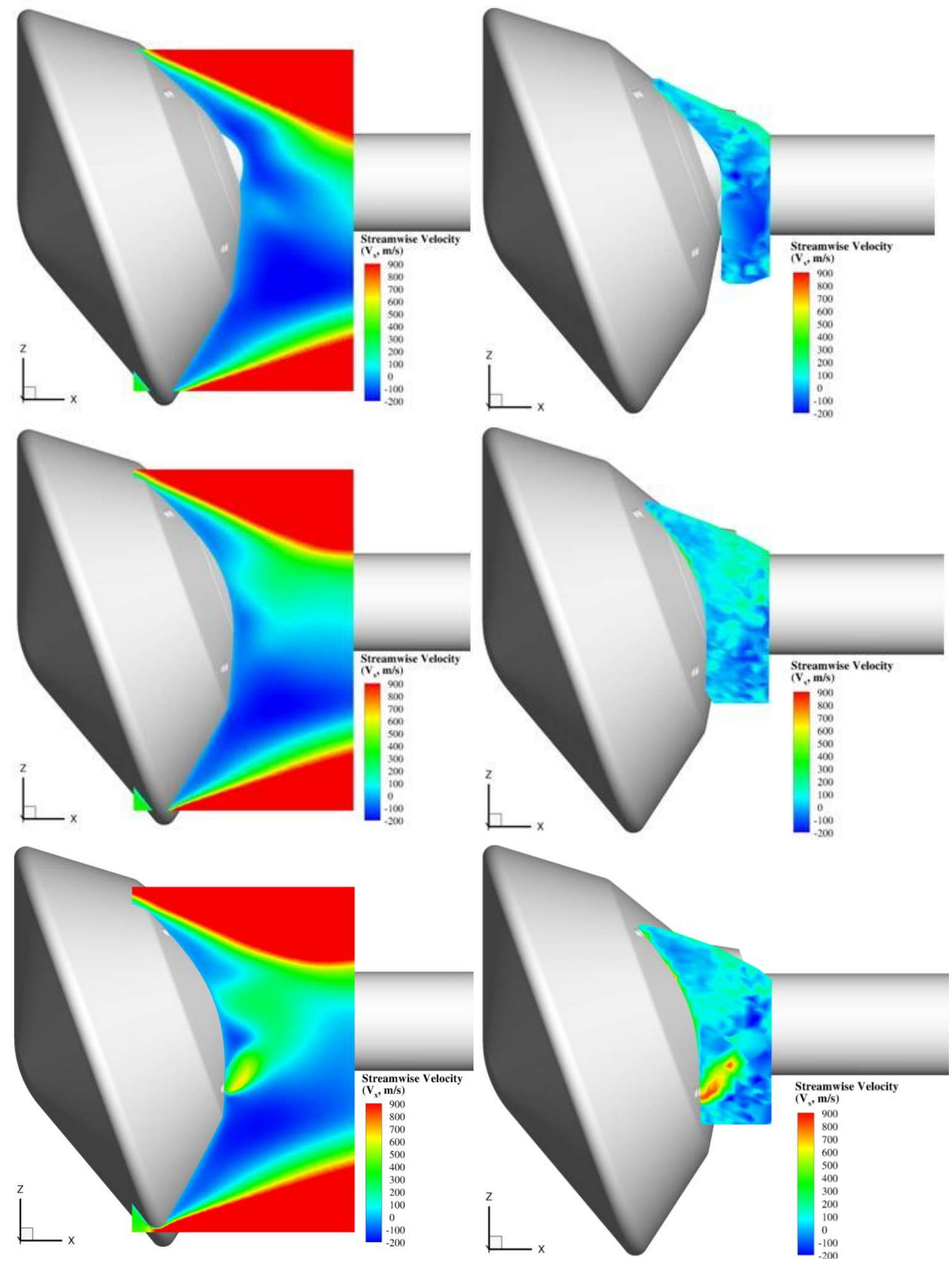

Figure A3. Contour map of CFD predicted (left) and experimentally measured (right) streamwise velocity $\left(V_{x}\right)$. Laser sheet positioned at $y=-20 \mathrm{~mm}\left(1^{\text {st }}\right.$ row $),-26 \mathrm{~mm}\left(2^{\text {nd }}\right.$ row $)$, and $-32.5 \mathrm{~mm}\left(3^{\text {rd }}\right.$ row $)$ from the model centerline. RCS jet is activated. 


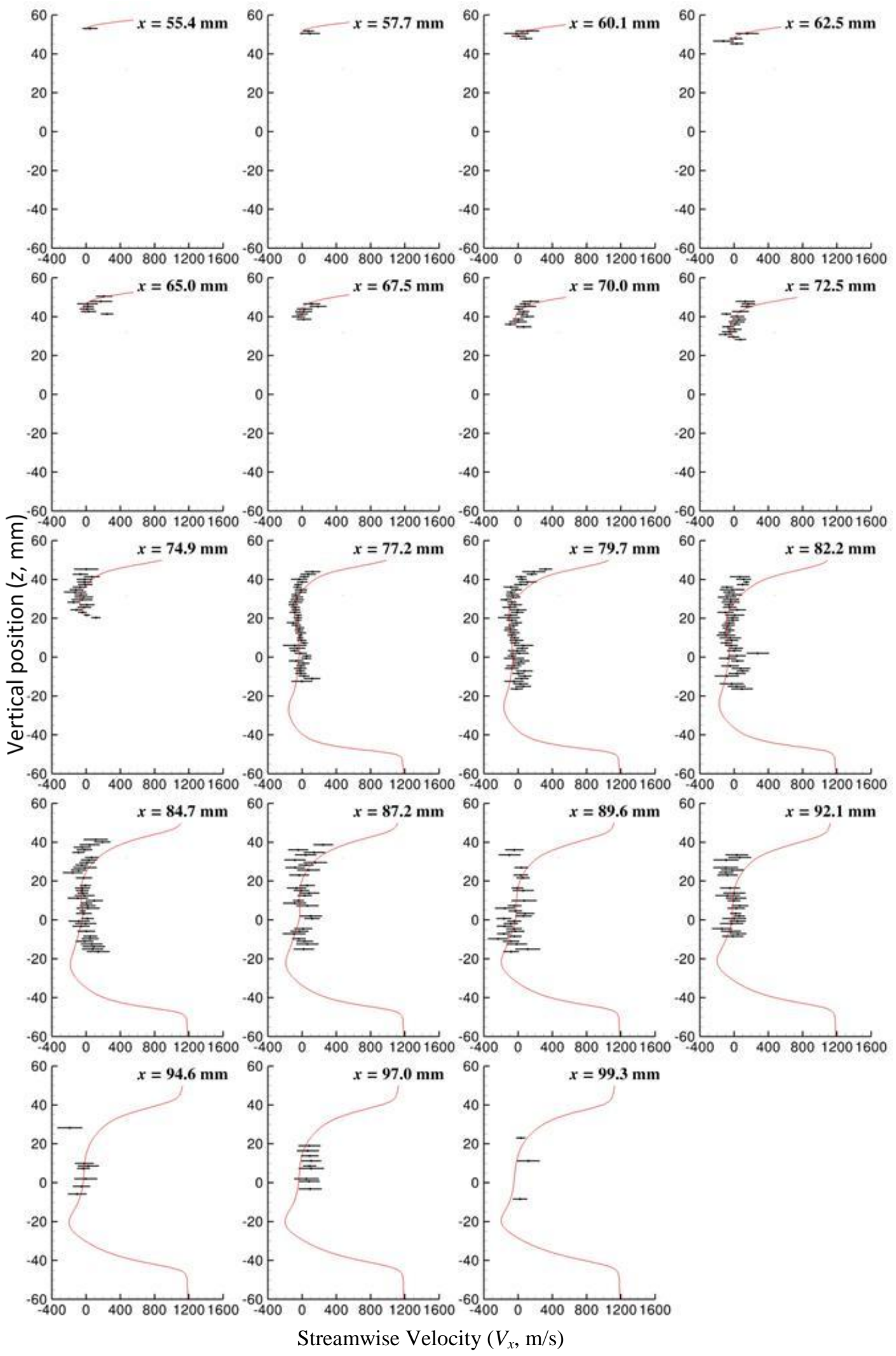

Figure A4. Vertical $(z)$ distribution of streamwise velocity $\left(V_{x}\right)$ at $y=-26 \mathrm{~mm}$. RCS jet is deactivated. Width of bars indicates uncertainty in the mean values. 

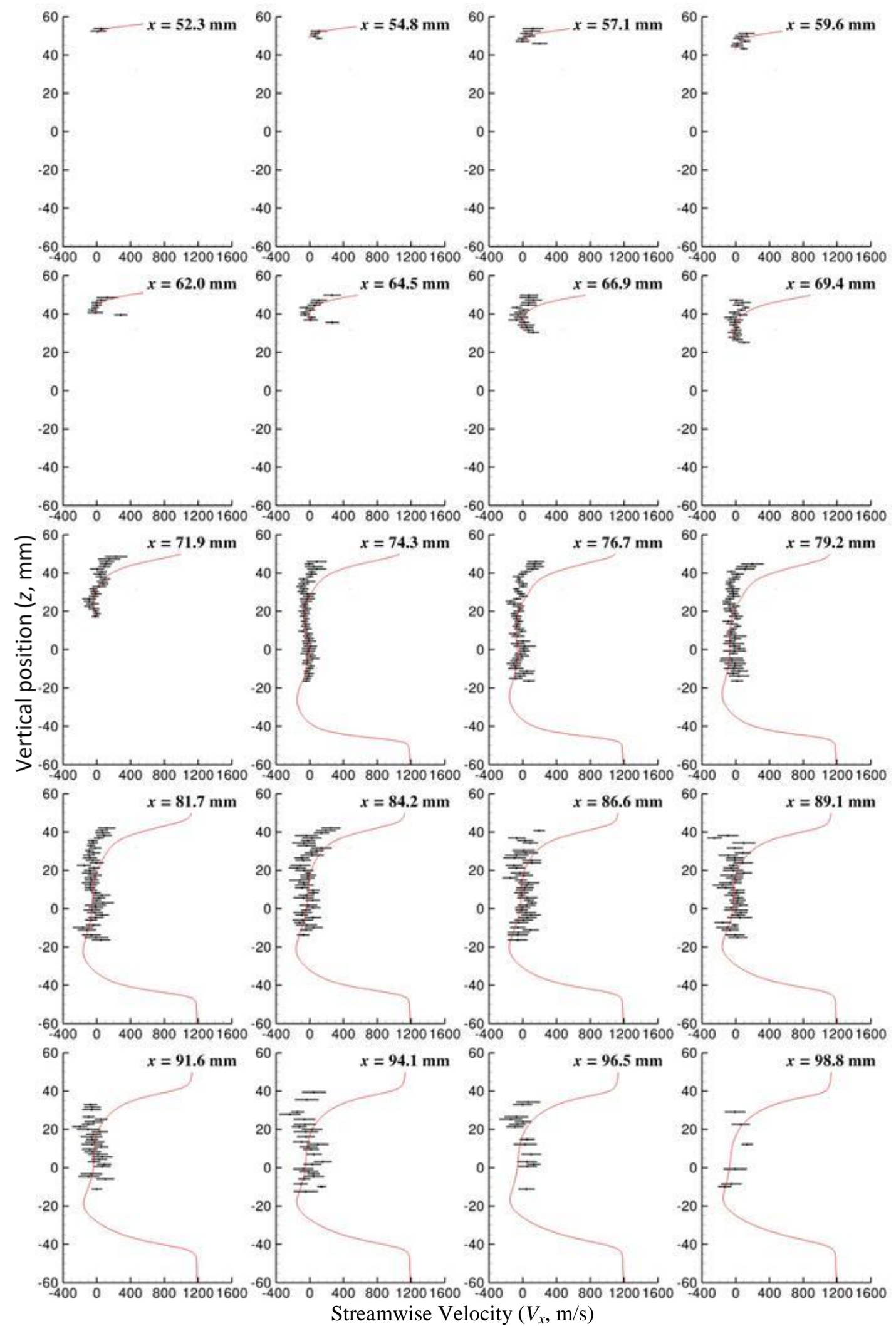

Figure A5. Vertical $(z)$ distribution of streamwise velocity $\left(V_{x}\right)$ at $y=-32.5 \mathrm{~mm}$. RCS jet is deactivated. Width of bars indicates uncertainty in the mean values.

American Institute of Aeronautics and Astronautics 

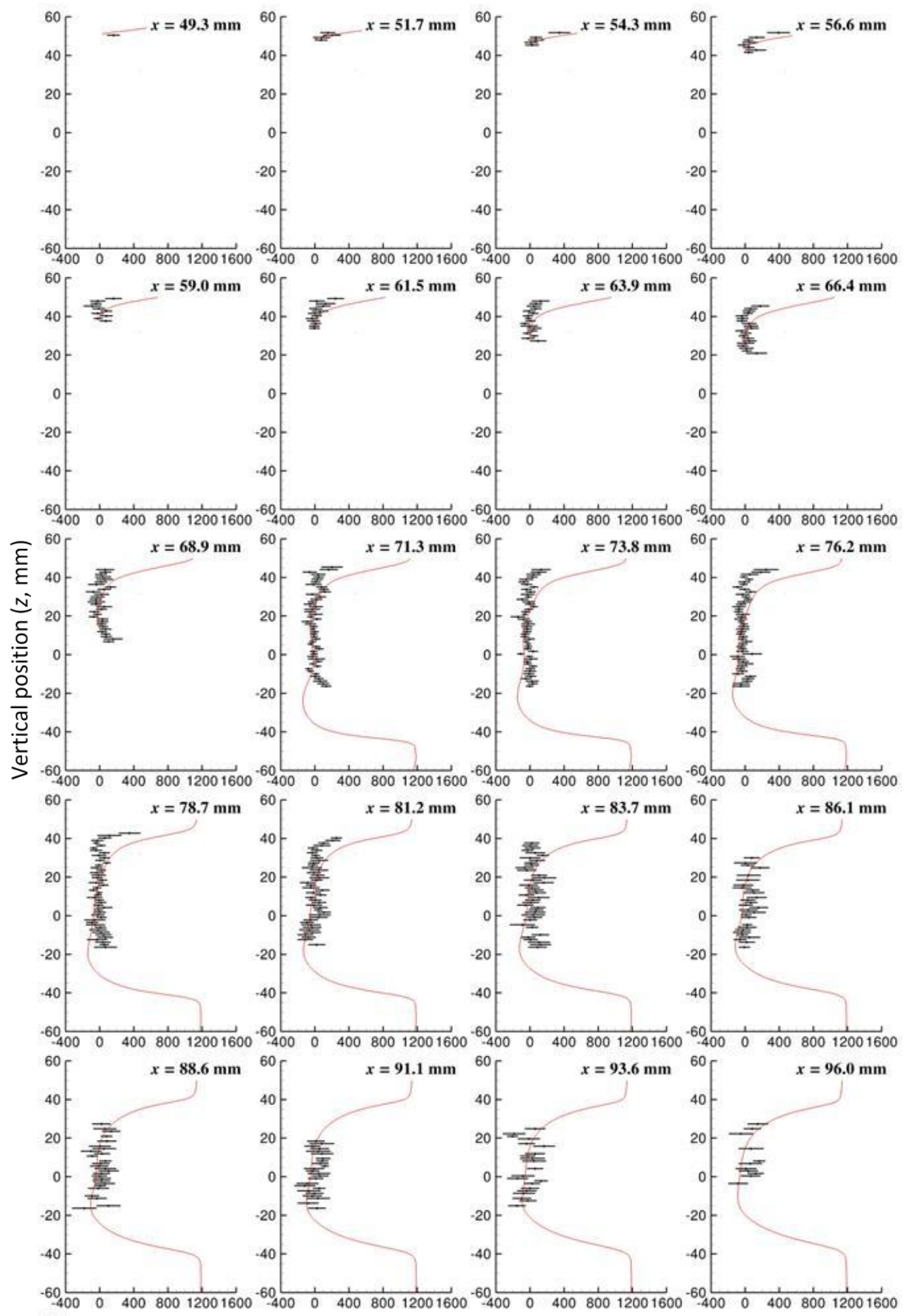

Streamwise Velocity $\left(V_{x}, \mathrm{~m} / \mathrm{s}\right)$

Figure A6. Vertical (z) distribution of streamwise velocity $\left(V_{x}\right)$ at $y=-39 \mathrm{~mm}$. RCS jet is deactivated. Width of bars indicates uncertainty in the mean values. 

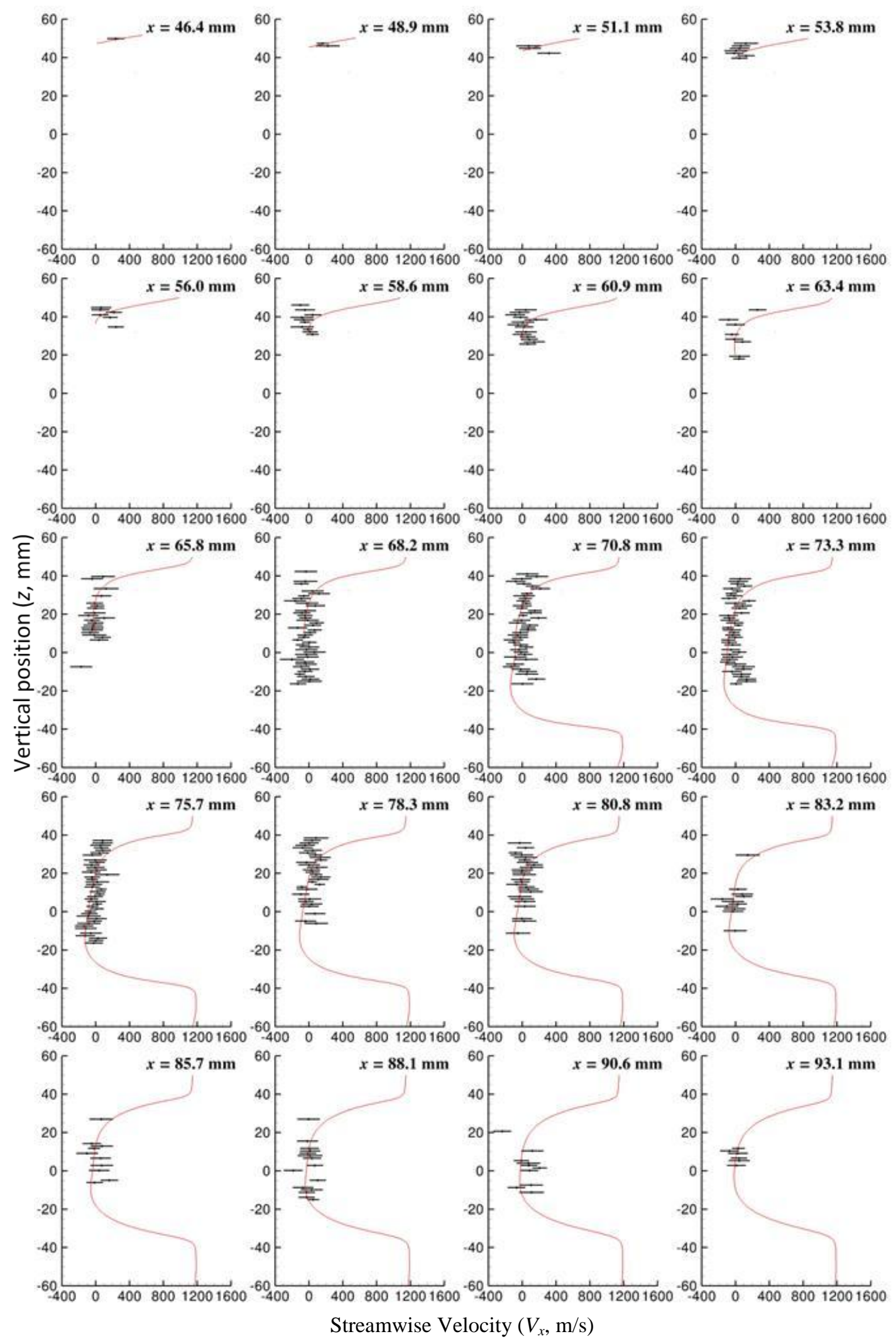

Figure A7. Vertical $(z)$ distribution of streamwise velocity $\left(V_{x}\right)$ at $y=-45 \mathrm{~mm}$. RCS jet is deactivated. Width of bars indicates uncertainty in the mean values. 


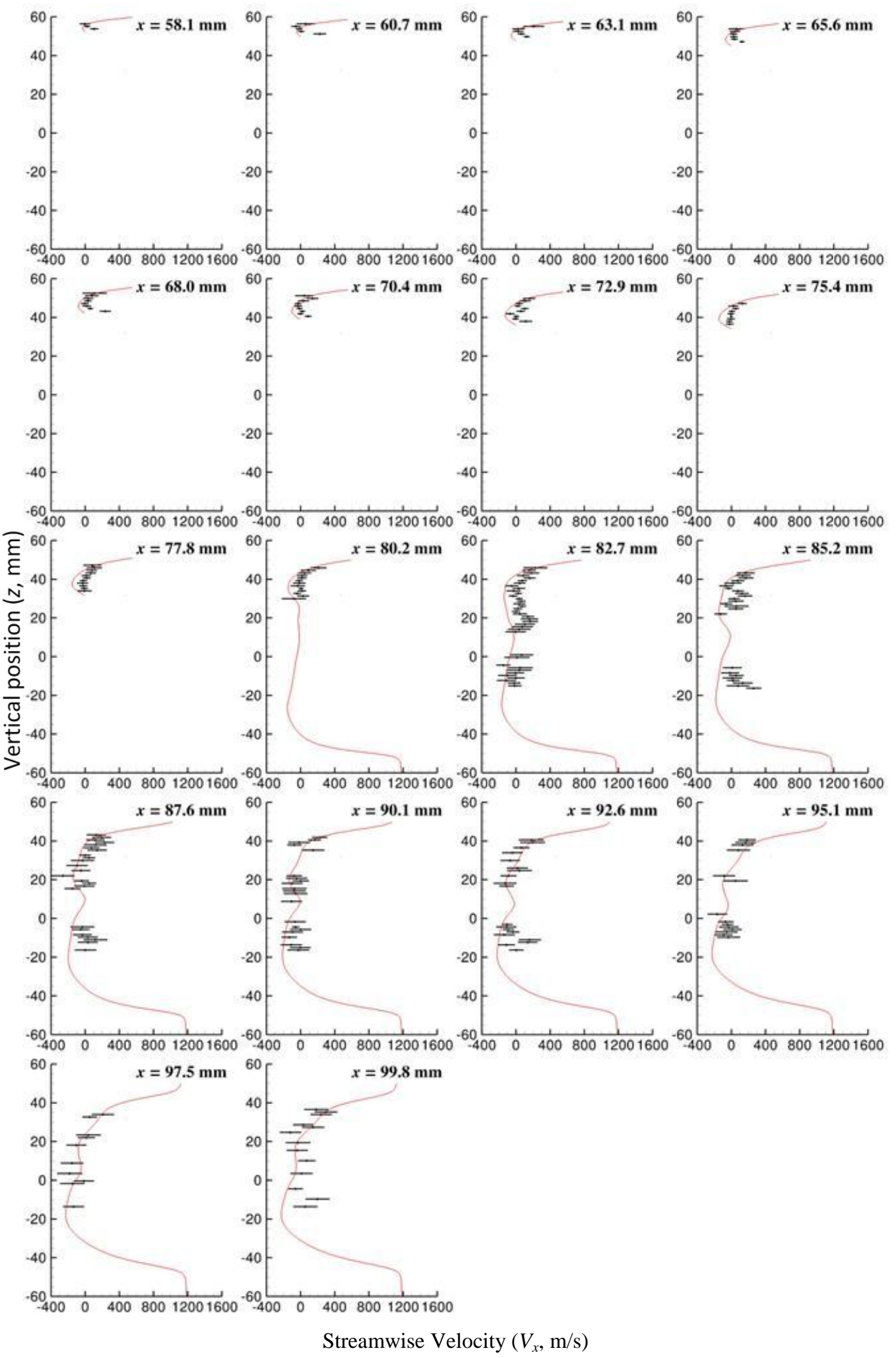

Figure A8. Vertical (z) distribution of streamwise velocity $\left(V_{x}\right)$ at $y=-20 \mathrm{~mm}$. RCS jet is activated. Width of bars indicates uncertainty in the mean values.

American Institute of Aeronautics and Astronautics 


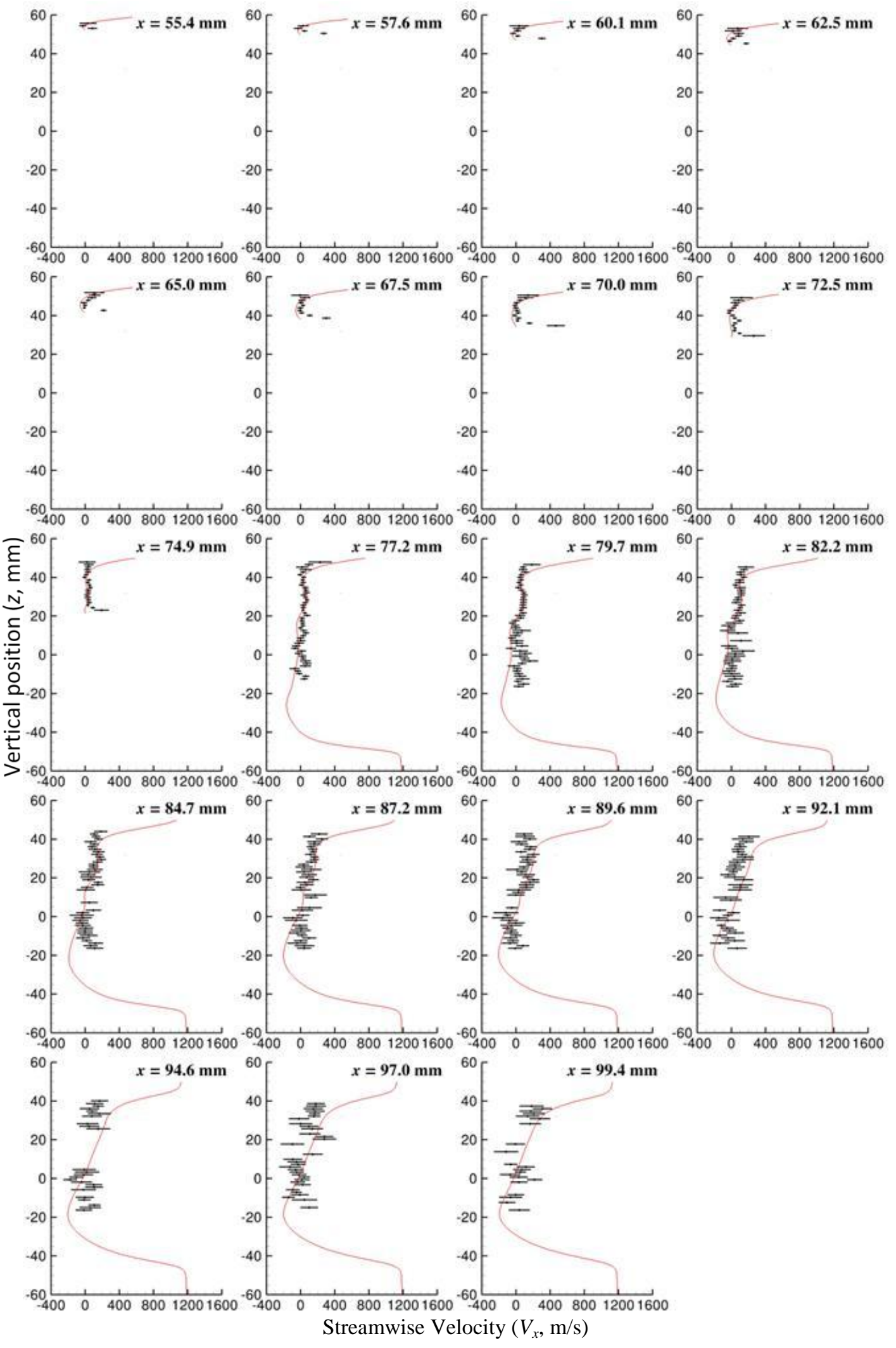

Figure A9. Vertical (z) distribution of streamwise velocity $\left(V_{x}\right)$ at $y=-26 \mathrm{~mm}$. RCS jet is activated. Width of bars indicates uncertainty in the mean values. 

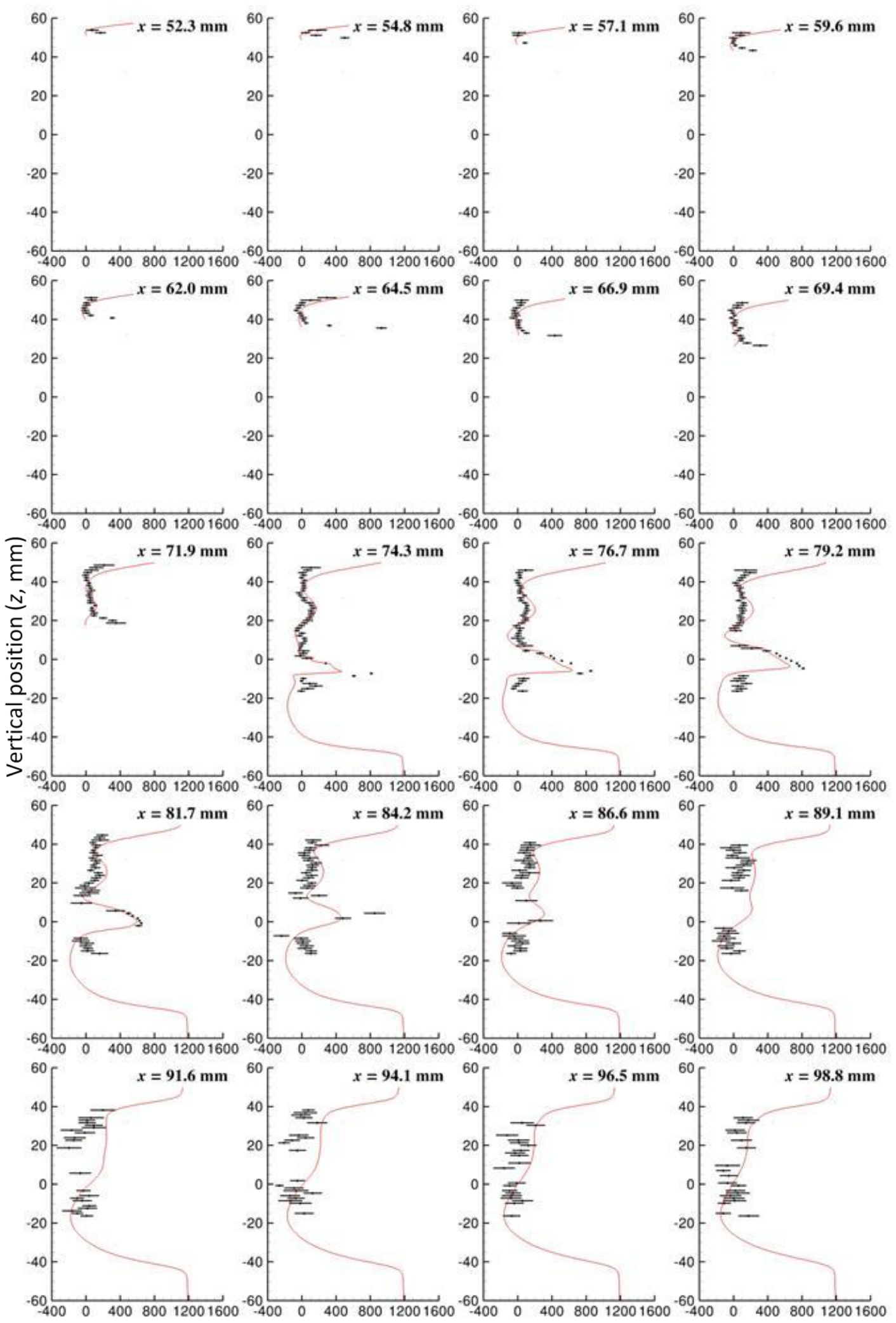

Streamwise Velocity $\left(V_{x}, \mathrm{~m} / \mathrm{s}\right)$

Figure A10. Vertical $(z)$ distribution of streamwise velocity $\left(V_{x}\right)$ at $y=-32.5 \mathrm{~mm}$. RCS jet is activated. Width of bars indicates uncertainty in the mean values. 

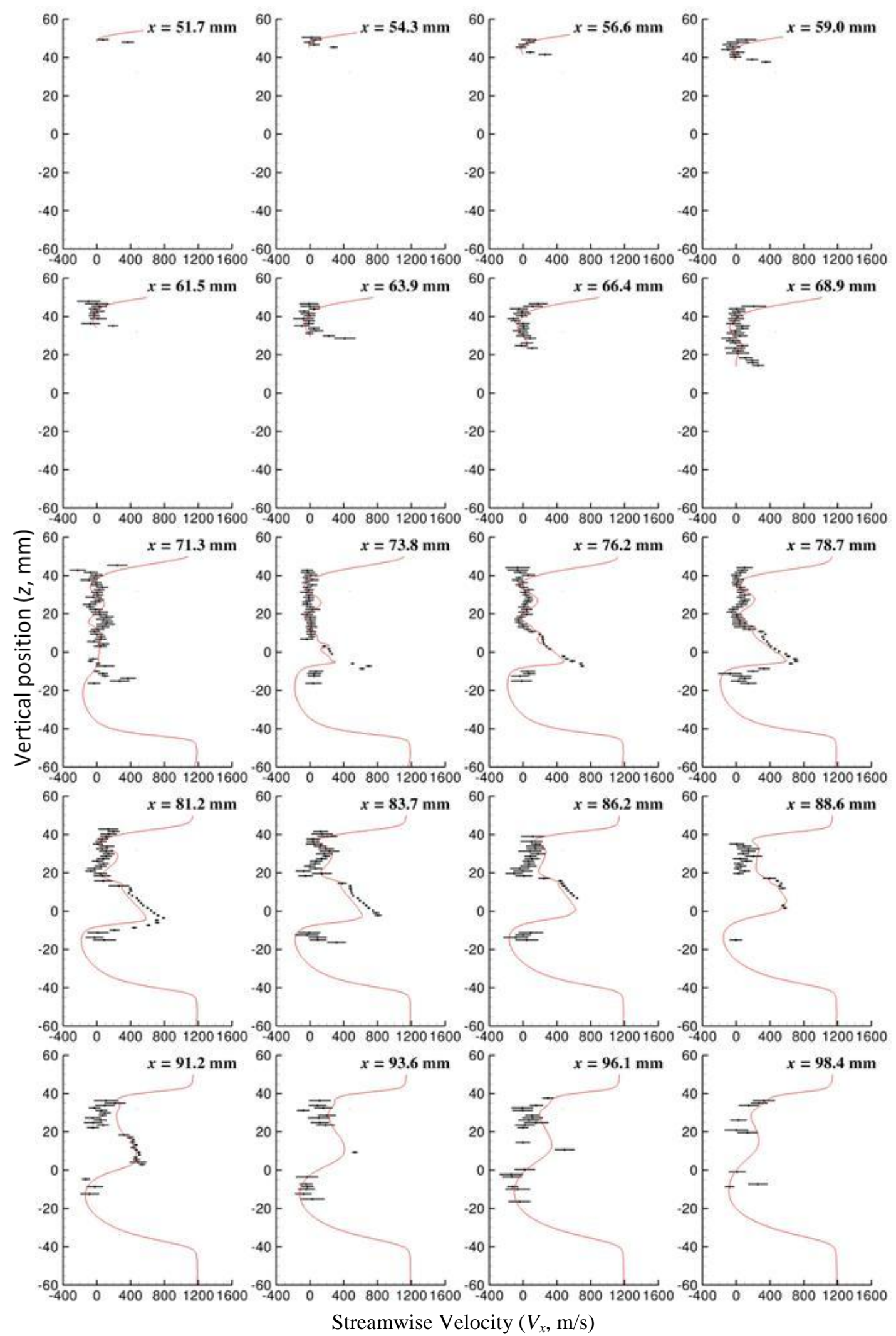

Figure A11. Vertical (z) distribution of streamwise velocity $\left(V_{\mathrm{x}}\right)$ at $y=-39 \mathrm{~mm}$. RCS jet is activated. Width of bars indicates uncertainty in the mean values. 